\title{
On the coverage of neutralino dark matter in coannihilations at the upgraded LHC
}

\author{
Murat Abdughani ${ }^{1,2,3, a}$, Lei Wu ${ }^{1, b}$ \\ ${ }^{1}$ Department of Physics, Institute of Theoretical Physics, Nanjing Normal University, Nanjing 210023, China \\ ${ }^{2}$ CAS Key Laboratory of Theoretical Physics, Institute of Theoretical Physics, Chinese Academy of Sciences, Beijing 100190, China \\ ${ }^{3}$ School of Physics, University of Chinese Academy of Sciences, Beijing 100049, China
}

Received: 29 October 2019 / Accepted: 1 March 2020 / Published online: 12 March 2020

(C) The Author(s) 2020

\begin{abstract}
In the supersymmetric models, the coannihilation of the neutralino DM with a lighter supersymmetric particle provides a feasible way to accommodate the observed cosmological DM relic density. Such a mechanism predicts a compressed spectrum of the neutralino DM and its coannihilating partner, which results in the soft final states and makes the searches for sparticles challenging at colliders. On the other hand, the abundance of the freeze-out neutralino DM usually increases as the DM mass becomes heavier. This implies an upper bound on the mass of the neutralino DM. Given these observations, we explore the HE-LHC coverage of the neutralino DM for the coannihilations. By analyzing the events of the multijet with the missing transverse energy $\left(E_{T}^{m i s s}\right.$ ), the monojet, the soft lepton pair plus $E_{T}^{\text {miss }}$, and the monojet plus a hadronic tau, we find that the neutralino DM mass can be excluded up to 2.6, 1.7 and $0.8 \mathrm{TeV}$ in the gluino, stop and wino coannihilations at the $2 \sigma$ level, respectively. However, there is still no sensitivity of the neutralino DM in stau coannihilation at the HE-LHC, due to the small cross section of the direct stau pair production and the low tagging efficiency of soft tau from the stau decay.
\end{abstract}

\section{Contents}

1 Introduction . . . . . . . . . . . 1

2 Coannihilating neutralino DM . . . . . . . 2

3 Prospects for coannihilations at the HE-LHC . . . . 3

3.1 Gluino coannihilation . . . . . . . . . . . 4

3.2 Stop coannihilation . . . . . . . . . . . 6

3.3 Wino coannihilation . . . . . . . . . . . 8

3.4 Stau coannihilation . . . . . . . . . . . 10

4 Conclusions . . . . . . . . . . . . . . 12

References . . . . . . . . . . . . . . . 13

a e-mail: mulati@mail.itp.ac.cn

b e-mail: leiwu@njnu.edu.cn (corresponding author)

\section{Introduction}

The presence of dark matter (DM) in the Universe has been established by versatile astrophysical and cosmological observations. However, its nature still remains a mystery. Weakly Interacting Massive Particles (WIMPs) are among the compelling dark matter candidates, whose mass is basically in the range from about $2 \mathrm{GeV}$ [1] up to several 100 $\mathrm{TeV}$ [2] and interaction strengthes are of the order of weak coupling of the Standard Model. As the WIMP DM can naturally produce the measured thermal relic density, there have been many experiments devoting into the searches of WIMP DMs [3].

In the minimal supersymmetric standard model (MSSM), the lightest neutralino $\tilde{\chi}_{1}^{0}$ can serve as the WIMP DM candidate when the $R$-parity is conserved. According to the composition of $\tilde{\chi}_{1}^{0}$, it can be bino-like, wino-like, higgsino-like or the mixed state. If $\tilde{\chi}_{1}^{0}$ is wino-like or higgsino-like state, their annihilation rates are large so that their masses have to be in $\mathrm{TeV}$ region to saturate the observed $\mathrm{DM}$ relic density [4]. On the other hand, if $\tilde{\chi}_{1}^{0}$ is bino-like state, its interaction with the SM particles are weak, which usually leads to an overabundance of DM. Among ways to solve this problem, coannihilation of $\tilde{\chi}_{1}^{0}$ with a light sparticle is an interesting mechanism [5]. Several coannihilation scenarios have been studied in supersymmetric models, such as the constrained MSSM (CMSSM) or mSUGRA, where the large negative value of $A_{0}$ drives the lighter stop or stau to be degenerate with the neutralino LSP, and the relic density of $\tilde{\chi}_{1}^{0}$ is brought into the range allowed by coannihilation with the stop or stau [6-12]. Besides, in the MSSM with non-universal gaugino masses or the vector-like extension of the MSSM, the gauginos can be the NLSP with a mass sufficiently close to that of the LSP so that gaugino coannihilation becomes important [13-21]. 
Coannihilating DM usually has some distinctive phenomenologies in DM experiments. For example, with the development of low noise technique and the increasing volume of detector, the sensitivity of DM direct detection experiment has been greatly improved. The null results produce strong constraints on various WIMP DM models. In coannihilation, due to the nature of bino, the neutralino DM weakly interacts with the quarks so that it can escape the bounds from the direct detection experiments.

Besides, there have been a great efforts devoted to searching for sparticles at the LHC. The colored sparticles have been excluded up to $\mathrm{TeV}$ in simplified models. However, these exclusion limits in the compressed regions will become weak, and even vanish. It should be mentioned that various compressed SUSY mass spectra have been also studied in LHC experiments. For example, the mass difference of compressed electroweakinos (such as bino-wino coannihilation scenario) can be probed down to about $2 \mathrm{GeV}$ by the current ATLAS search of two soft leptons plus missing transverse energy events [22]. However, when the mass difference between the LSP and NLSP is of the order of the rho meson or below (but is still larger than about $400 \mathrm{MeV}$ ), ${ }^{1}$ there is still no limit at the LHC. It should be mentioned that the future hadron colliders are hardly to improved the sensitivity in operate at a center of mass energy $\sqrt{s}=27 \mathrm{TeV}$, and to collect of the order of $15 \mathrm{ab}^{-1}$ of data during 20 years of operation, which can greatly extend the HL-LHC potential of accessing the mass ranges of new particles [39,40]. In Ref. [41], the authors investigated for observability of $\mathrm{TeV}$ higgsino and wino-like neutralino DMs at the HE-LHC. In Ref. [42], the authors studied the phenomenology of stop, gluino and higgsinos in natural SUSY at the LHC. Other studies of the discovery reach of supersymmetric particles at the HELHC can be found in [43-48]. In this paper, we will explore prospects for the coverage of neutralino dark matter coannihilation at the HE-LHC. We begin to identify the parameter space of stop, gluino, wino and stau coannihilation under the LHC and DM constraints in Sect. 2, and then perform the detailed Monte Carlo (MC) simulation for each coannihilation scenario at the HE-LHC in Sect. 3. Finally, we draw conclusions in Sect. 4.

\section{Coannihilating neutralino DM}

After the electroweak symmetry is broken in the MSSM, the mass matrix for neutralinos in $\operatorname{bino}(\tilde{B})$-wino $(\tilde{W})$-higgsinos $\left(\tilde{H}_{u, d}^{0}\right)$ basis can be written as

$M_{\tilde{\chi}^{0}}=\left(\begin{array}{cccc}M_{1} & 0 & -\cos \beta \sin \theta_{w} m_{Z} & \sin \beta \sin \theta_{w} m_{Z} \\ 0 & M_{2} & \cos \beta \cos \theta_{w} m_{Z} & \sin \beta \cos \theta_{w} m_{Z} \\ -\cos \beta \sin \theta_{w} m_{Z} & \cos \beta \cos \theta_{w} m_{Z} & 0 & -\mu \\ \sin \beta \sin \theta_{w} m_{Z} & -\sin \beta \cos \theta_{w} m_{Z} & -\mu & 0\end{array}\right)$

these compressed regions because of the low reconstruction efficiency of soft leptons and contamination of high pile-up [24].

In coannihilation, the mass difference between the neutralino DM and its coannihilating partner is usually small. Consequently, the light sparticles in coannihilation can be still consistent with the LHC data. Another interesting fact is that the abundance of neutralino DM in the freeze-out mechanism usually increases as the mass of the neutralino DM becomes heavier. This leads to the upper limits of the neutralino DM and its coannilating partner masses, which provides a guideline of searching for supersymmetry at colliders. Therefore, it is interesting to explore various coannihilation scenarios at the LHC and future colliders [25-37].

Beyond the LHC, the high-energy LHC (HE-LHC) is proposed to built on current LHC tunnel by upgrading to $16 \mathrm{~T}$ superconducting magnet [38]. The HE-LHC is designed to

\footnotetext{
${ }^{1}$ If the mass splitting is less than about $400 \mathrm{MeV}$, the disappearing track search can be used to investigate are such almost degenerate electroweakinos [23].
}

where $M_{1}$ and $M_{2}$ are $U(1)_{Y}$ and $S U(2)_{L}$ soft supersymmetry breaking mass parameters, respectively. $\mu$ is the higgsino mass parameter and $\theta_{w}$ is the weak mixing angle. We can diagonalize the Eq. (2.1) by a unitary $4 \times 4$ matrices $N_{i j}$ [49], and then have the mass eigenstates $\tilde{\chi}_{1,2,3,4}^{0}$. When the $R$-parity is conserving, the lightest neutralino $\tilde{\chi}_{1}^{0}$ can play the role of the DM and provide the correct relic density by itself. However, if there exists other sparticles whose masses are nearly degenerate with $\tilde{\chi}_{1}^{0}$, the relic abundance of the neutralino DM is determined not only by its annihilation cross section, but also by the annihilation of these heavier sparticles. This case is, namely, coannihilation. The effective coannihilation cross section can be written as [50],

$$
\begin{aligned}
\sigma_{\text {eff }}= & \frac{g_{\tilde{\chi}_{1}^{0}}^{2}}{g_{\text {eff }}^{2}}\left\{\sigma_{\tilde{\chi}_{1}^{0} \tilde{\chi}_{1}^{0}}+2 \sigma_{\tilde{\chi}_{1}^{0}} \mathcal{P} \frac{g_{\mathcal{P}}}{g_{\tilde{\chi}_{1}^{0}}}(1+\Delta)^{3 / 2} \exp (-x \Delta)\right. \\
& \left.+\sigma_{\mathcal{P} \mathcal{P}} \frac{g_{\mathcal{P}}^{2}}{g_{\tilde{\chi}_{1}^{0}}^{2}}(1+\Delta)^{3} \exp (-2 x \Delta)\right\} .
\end{aligned}
$$

Here $\Delta=\left(m_{\mathcal{P}}-m_{\tilde{\chi}_{1}^{0}}\right) / m_{\tilde{\chi}_{1}^{0}}$ and $x=m_{\tilde{\chi}_{1}^{0}} / T$. The parameters $g_{\tilde{\chi}_{1}^{0}}$ and $g_{\mathcal{P}}$ are the numbers of degrees of freedom of 
DM and coannilating partner $\mathcal{P}$, respectively. The effective coupling $g_{\text {eff }}$ is given by,

$g_{\text {eff }}=g_{\tilde{\chi}_{1}^{0}}+g_{\mathcal{P}}(1+\Delta)^{3 / 2} \exp (-x \Delta)$.

From Eq. 2.2, it can be seen that the contributions of the terms including $\sigma_{\tilde{\chi}_{1}^{0} \mathcal{P}}$ and $\sigma_{\mathcal{P} \mathcal{P}}$ can become important, even dominant over $\sigma_{\tilde{\chi}_{1}^{0}}^{0} \tilde{x}_{1}^{0}$, when $\Delta$ is vanishing.

We will carry out our study of coannihilations in the simplified MSSM, where only relevant sparticles in each scenario are involved. Such a framework allows us to remain agnostic of the detailed UV-physics, yet still capture the feature of coannihilation. We scan the ranges of SUSY mass parameters in gluino, stop, wino and stau coannihilations as following:

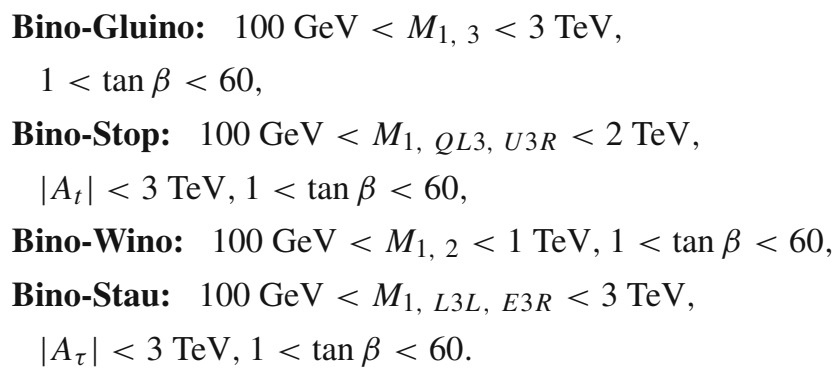

In above each scenario, we assume the $\mathrm{CP}$ odd Higgs mass $m_{A}$ and other soft SUSY breaking mass parameters as a common value $M_{S U S Y}=5 \mathrm{TeV}$, and take other irrelevant trilinear soft SUSY breaking parameter $A=0$. We calculate the DM relic density $\Omega_{\tilde{\chi}} h^{2}$ with MicrOMEGAs [51] and the Higgs mass with SUSY-HIT [52]. We require our samples to satisfy the $2 \sigma$ bounds of the Planck value of DM relic density [53] and the measured Higgs mass within the range of $125 \pm 3 \mathrm{GeV}$ [54]. In addition, we impose the vacuum stability constraints in stop and stau coannihilations, since the large mixing in stop and stau sector may lead to the charge or color breaking [55-57]. Besides, we also consider the experimental 95\% C.L. exclusion limits from the null results of the LHC searches for gluino [58], stop [59] and wino [22], which are calculated by using $C L_{s}$ prescription with the expected and observed number of events at the experiment.

In Fig. 1, we show the mass ranges of the neutralino DM and its coannihilating partners for the surviving samples allowed by the DM relic density, the Higgs mass and the vacuum stability conditions for each coannihilation. As known, when the neutralino DM becomes heavy, the abundance of the neutralino DM will overclose the Universe. This leads to an upper limit of the neutralino DM mass. Among these scenarios, the gluino and stop interact strongly and extend the allowed mass range of the LSP for accommodating the correct relic density, while the wino and stau interact weakly and have to be lighter. From the Fig. 1, we can see that the neutralino DM and stop masses in the stop coan- nihilation should be lighter than $1.9 \mathrm{TeV}$. The mass difference between the neutralino DM and the stop varies from 2 to $40 \mathrm{GeV}$. Most of samples are right-handed stop, which will decay through the loop process $\tilde{t}_{1} \rightarrow \tilde{\chi}_{1}^{0}+c$ and fourbody process $\tilde{t}_{1} \rightarrow \tilde{\chi}_{1}^{0}+b f \bar{f}^{\prime}$. In the gluino coannihilation, the upper limit of the neutralino DM mass is about 3 $\mathrm{TeV}$, which is much greater than that in other three scenarios, because the gluino-gluino annihilation has a larger cross section. For the same reason, the maximal mass difference between the neutralino DM and the gluino can reach about $100 \mathrm{GeV}$. Such a gluino mainly decays through the process $\tilde{g} \rightarrow \tilde{\chi}_{1}^{0} q \bar{q}$. While for the wino coannihilation, the neutralino $\mathrm{DM}$ and the next-to-lightest neutralino $\tilde{\chi}_{2}^{0}$ masses are less than $900 \mathrm{GeV}$. Due to their small mass splittings, the coannihilating partners will dominantly decay to the SM particles via $\tilde{\chi}_{2}^{0} \rightarrow Z^{*} \rightarrow \tilde{\chi}_{1}^{0} f \bar{f}$ and $\tilde{\chi}_{1}^{ \pm} \rightarrow W^{ \pm *} \rightarrow \tilde{\chi}_{1}^{0} f \bar{f}^{\prime}$. As for stau coannihilation, the main contribution to the relic density comes from stau-stau annihilation into $\tau^{+} \tau^{-}$for light stau, and into $h h, Z Z$ and $W^{ \pm} W^{\mp}$ for heavy stau. Besides, in Fig. 1, we can also see that the null results of the LHC searches for sparticles have produced the lower bounds on gluino, stop and wino masses in coannihilation regions, that is, $m_{\tilde{t}_{1}}>400 \mathrm{GeV}, m_{\tilde{g}}>1000 \mathrm{GeV}$ and $m_{\tilde{\chi}_{2}^{0}}>150 \mathrm{GeV}$. While there is still no stronger limit on the stau mass from the LHC data than that from the LEP experiment.

Due to the Sommerfeld enhancement effects at low velocities, the coannihilation rates can be increased so that the upper bounds of the neutralino DM masses will be altered [60-68]. For example, the bino-like neutralino DM mass in stop coannihilation consistent with the observed DM abundance turns out to be several TeV $[9,69]$. Besides the Sommerfeld enhancement, the bound-state effects [70-74] and the higher order perturbative corrections [75-79] can further increase the neutralino DM coannihilation rates and thus extend the neutralino DM mass that can give the observed DM relic density. Given the designed colliding energy of the HE-LHC, we do not include those two effects in our calculations because the following results of their masses reach will not be changed. Besides, it should be noted that the decay widths of the coannihilating partners will become small when their masses are very close the neutralino DM. In this case those sparticles will have a long life in the detector. We leave the detailed analysis of searching for the long lived sparticles in our future work.

\section{Prospects for coannihilations at the HE-LHC}

Next, we study the prospects of searching for these sparticles in coannihilations at the HE-LHC. The cross sections of our signal and background processes are calculated at LO. We simulated the signal and background events by the package MG5_aMC@NLO v2.6.1 [80] with the NN23LO1 

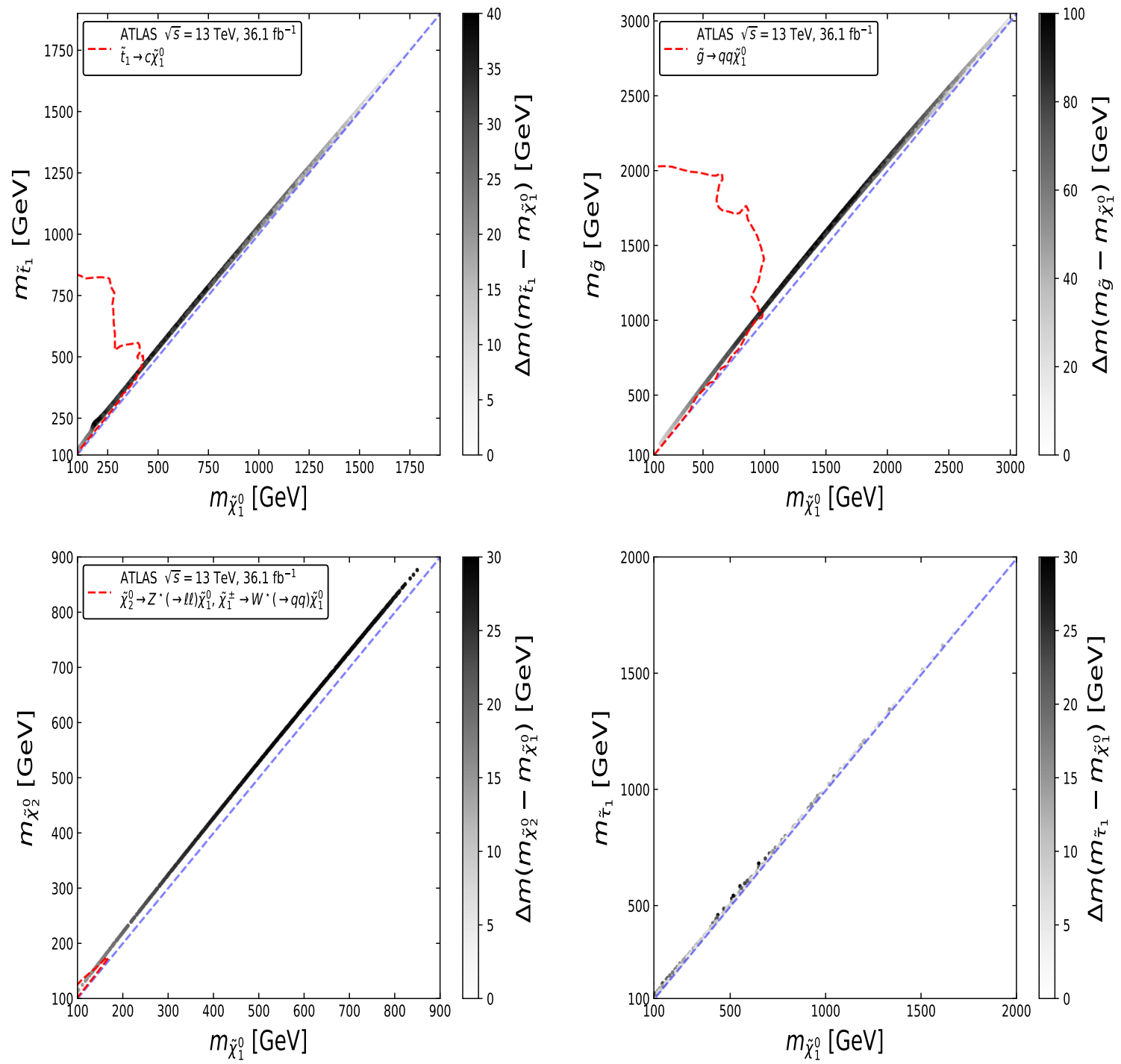

Fig. 1 The samples that satisfy the observed DM relic density, the Higgs mass and vacuum stability conditions are projected on the plane of DM mass and its coannihilating partner mass for bino-stop (top-left panel), bino-gluino (top-right panel), bino-wino (lower-left panel) and bino-stau (lower-right panel) coannihilations. The colormap represents

the mass difference of DM and coannihilating partner in each scenario. The red dashed lines denotes the 95\% C.L. exclusion limits from the null results of searching for gluino [58], stop [59] and wino [22] at the LHC-13 TeV with the luminosity $\mathcal{L}=36.1 \mathrm{fb}^{-1}$

PDF (Parton Distribution Function) set. Then the parton shower and hadronization are performed by the package Pythia8.230 [81]. The jets are clustered by using the anti$k_{t}$ algorithm [82] with the distance parameter $R=0.4$. We implement the fast detector simulation by the package Delphes3.4.1 [83]. It should be noted that the parton-level events of $Z / W+j e t s$ are generated up to two jets that are matched to the parton shower using the MLM-scheme with merging scale $Q=60 \mathrm{GeV}$. The event selections are carried out in the framework of CheckMATE-2.0.26 [84]. We evaluate the statistical significance with the formula $Z=S / \sqrt{B}$, where $S$ and $B$ denote the signal and background yields respectively.

\subsection{Gluino coannihilation}

The gluinos are mainly produced through two processes $g g \rightarrow \tilde{g} \tilde{g}$ and $q \bar{q} \rightarrow \tilde{g} \tilde{g}$ at the hadron colliders. In the gluino-neutralino coannihilation, the decay of the gluino is dominated by the three-body decay process $\tilde{g} \rightarrow q \bar{q} \tilde{\chi}_{1}^{0}$, and the subleading decay is the loop-induced process $\tilde{g} \rightarrow g \tilde{\chi}_{1}^{0}$ that is typically a few percent, which can be seen from Fig. 2 . Therefore, we use multi-jet plus missing transverse energy events from the process $p p \rightarrow \tilde{g} \tilde{g}$ with the sequent decay $\tilde{g} \rightarrow q \bar{q} \tilde{\chi}_{1}^{0}$ to probe the gluino coannihilation at the HELHC, as shown in Fig. 3. The main SM backgrounds include: $W(\rightarrow \ell v)+$ jets, $Z / \gamma^{\star}(\rightarrow \ell \bar{\ell})+$ jets, $\gamma+$ jets, $t \bar{t}$, single top, and dibosons $(W W, W Z, Z Z)$. 


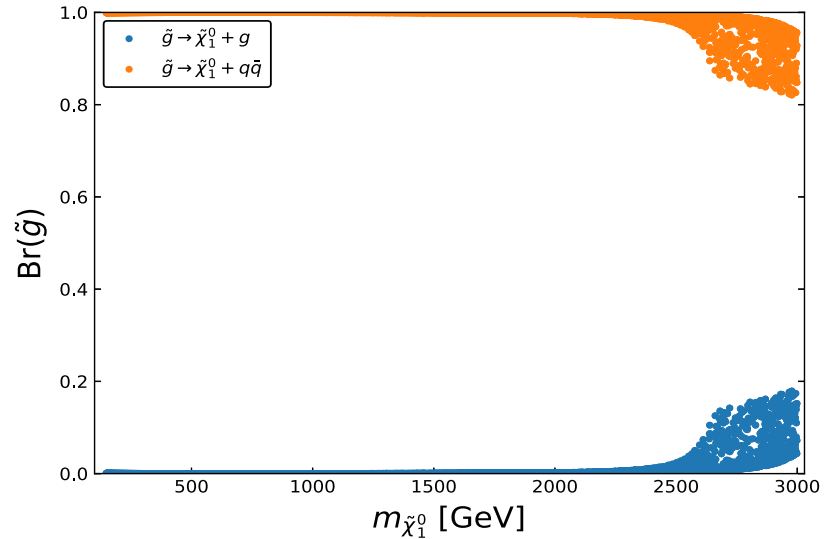

Fig. 2 The branching ratio of $\tilde{g}$ in the gluino coannihilation

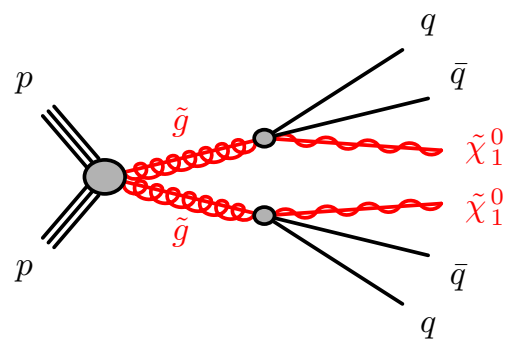

Fig. 3 The schematic diagram of the production process $p p \rightarrow \tilde{g} \tilde{g}$ with the sequent decay $\tilde{g} \rightarrow q \bar{q} \tilde{\chi}_{1}^{0}$ at the HE-LHC

In Fig. 5, we calculate the signal significance of the multijets plus missing transverse energy events from the process $p p \rightarrow \tilde{g} \tilde{g}$ for each surviving sample in the gluino coanni- hilation at the HE-LHC. From Fig. 5, we can see that the significance becomes small with the increase of the gluino mass. The neutralino DM with a mass less than about $1.6 \mathrm{TeV}$ will be probed at $Z \geq 5 \sigma$ level at the HE-LHC with the luminosity $\mathcal{L}=300 \mathrm{fb}^{-1}$. Such a mass reach can be enhanced up to about $2.2 \mathrm{TeV}$ if the luminosity increases to $15 \mathrm{ab}^{-1}$. On the other hand, the HE-LHC will be able to exclude the neutralino DM mass $m_{\tilde{\chi}_{1}^{0}}$ in gluino coannihilation up to about $1.9 \mathrm{TeV}$ and $2.6 \mathrm{TeV}$ at $Z=2 \sigma$ level, respectively.

We firstly check the existing LHC analysis of searching for the 2-6 jets plus missing transverse energy events [58] to determine the sensitive signal regions. We find that our gluino coannihilation is sensitive to the signal regions with 2 jets in the final states. Then, we optimize the LHC analysis at the HE-LHC. Due to the small mass difference between the gluino and the neutralino LSP, we will make use of the presence of initial-state radiation (ISR) jets by requiring a higher $p_{T}$ threshold on the most energetic jet in the event. The signal and background events can be effectively separated by using two kinematical variables, the effective mass $m_{\text {eff }}($ inclu $)$ [85] and the ratio of $E_{T}^{\text {miss }} / \sqrt{H_{T}}$, where $m_{\text {eff }}($ inclu $)$ is the scalar sum of transverse momenta of all reconstructed jets with $p_{T}>50 \mathrm{GeV}$, and $H_{T}$ is the scalar sum of the transverse momenta of all reconstructed jets. From the Fig. 4, we can see that most of the signal events lies in the ranges of $m_{\text {eff }}($ inclu $)>700 \mathrm{GeV}$. However, the $Z j$ and $W j$ background events fall off fast, which sequently followed by the $t \bar{t}$ and $V V$ backgrounds. Besides, $m_{\text {eff }}($ inclu) can also strongly suppress the multijet background. Since a hard ISR jet will boost $E_{T}^{\text {miss }}$, the signal events predict a larger value
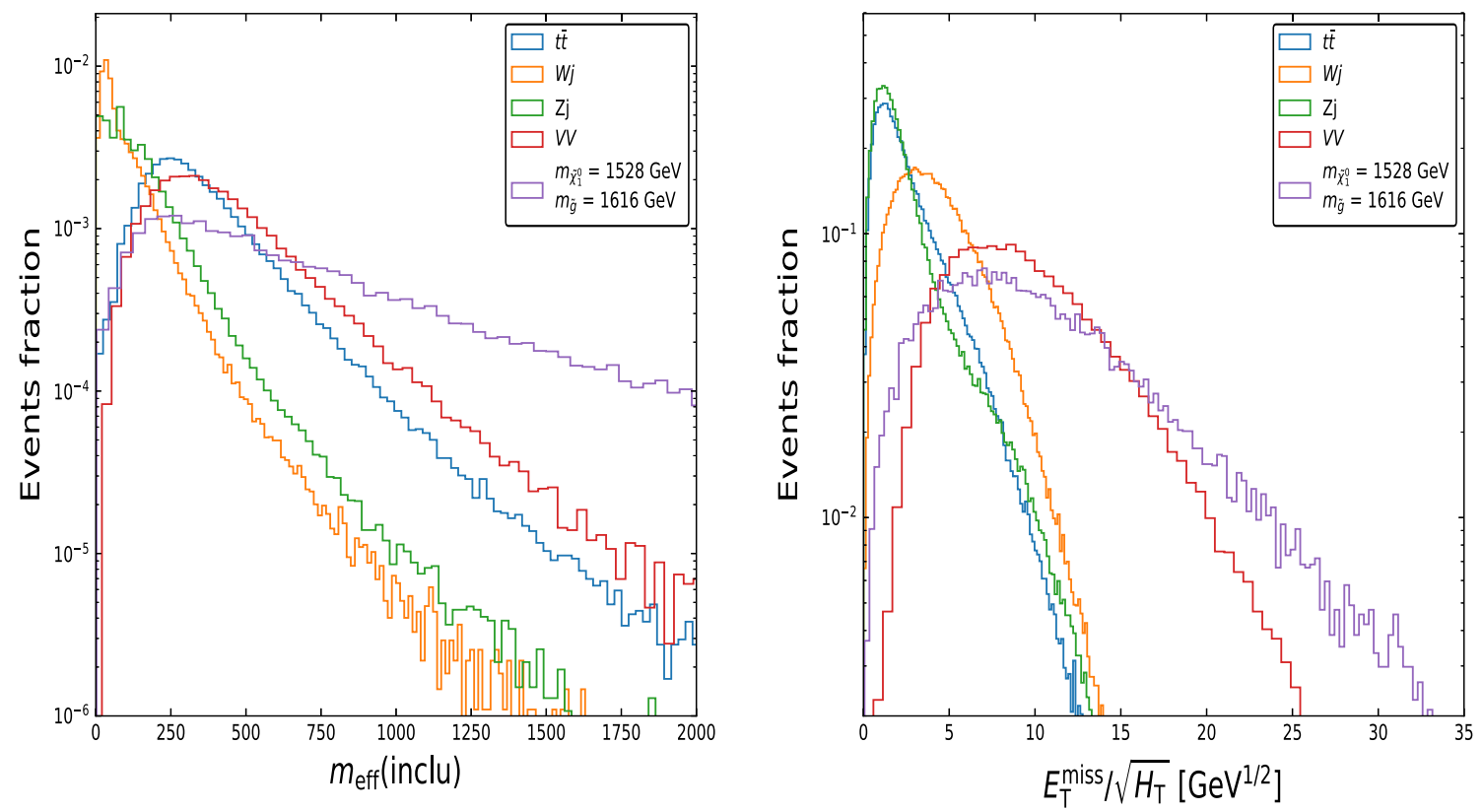

Fig. 4 The normalized distributions of $m_{\text {eff }}$ (inclu) and $E_{T}^{m i s s} / \sqrt{H_{T}}$ for the signal and background events at the HE-LHC. The benchmark point is $m_{\tilde{g}}=1616 \mathrm{GeV}$ and $m_{\tilde{\chi}_{1}^{0}}=1528 \mathrm{GeV}$ 
Table 1 Six signal regions are defined by the values of $p_{T}\left(j_{1}\right), m_{\text {eff }}($ inclu $)$ and $E_{T}^{\text {miss }} / \sqrt{H_{T}}$ in the multijet plus missing transverse energy events from the process $p p \rightarrow \tilde{g} \tilde{g}+X$ for the gluino coannihilation at the HE-LHC

\begin{tabular}{lrrrrr}
\hline SRs & $S R 1$ & $S R 2$ & $S R 3$ & $S R 4$ & SR5 \\
\hline$p_{\mathrm{T}}\left(j_{1}\right)(\mathrm{GeV})$ & 250 & 300 & 350 & 400 & 600 \\
$E_{\mathrm{T}}^{\text {miss }} / \sqrt{H_{\mathrm{T}}}\left(\mathrm{GeV}^{1 / 2}\right)>$ & 16 & 18 & 18 & 18 & 26 \\
$m_{\text {eff }}($ inclu $)(\mathrm{GeV})>$ & 1200 & 1600 & 2000 & 2400 & 160 \\
\hline
\end{tabular}

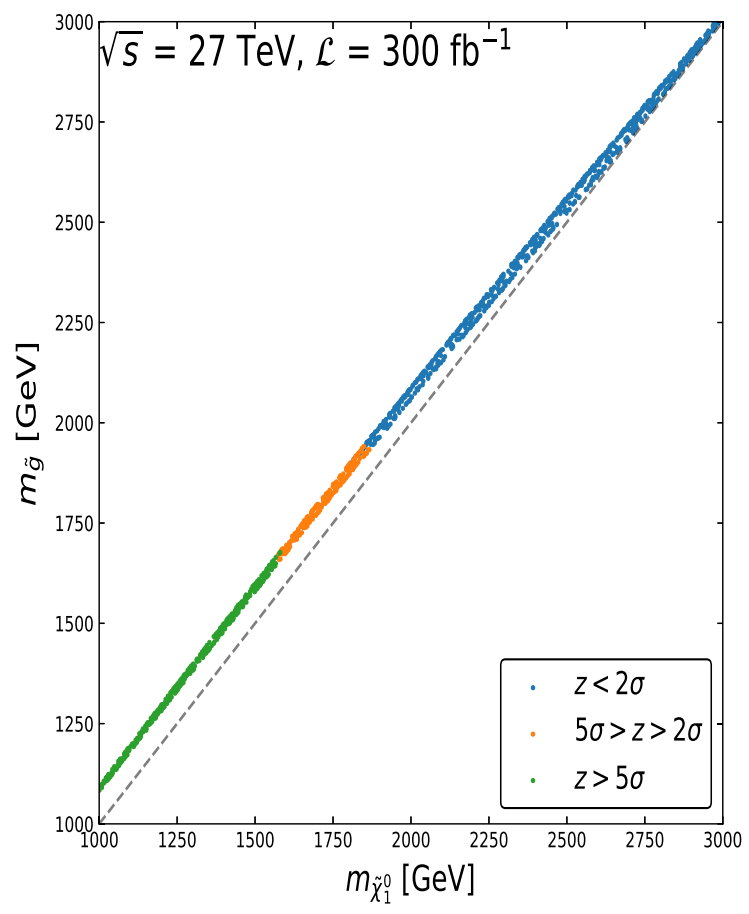

Fig. 5 The statistical significance $Z$ of the multijets plus missing transverse energy events from the process $p p \rightarrow \tilde{g} \tilde{g}+X$ for the gluino coannihilation at the HE-LHC with the integrated luminosity $\mathcal{L}=300 \mathrm{fb}^{-1}$

of $E_{T}^{\text {miss }} / \sqrt{H_{T}}$ than the background events. Such a cut can further enhance the sensitivity of our signal. Therefore, we impose the following event selection criteria:

- The Electrons and muons with $p_{T}>7 \mathrm{GeV}$ and $|\eta|<$ 2.47 are vetoed.

- At least two jets are required, where the leading jet has to satisfy $p_{T}\left(j_{1}\right)>200 \mathrm{GeV}$ and other jets should have $p_{T}\left(j_{i}\right)>50$, where $i>1$.

- The events are required to have $E_{T}^{\text {miss }}>250 \mathrm{GeV}$.

- The azimuthal angular distances between jets and missing enenrgy $\Delta \phi\left(j_{i}, p_{T}^{\text {miss }}\right)>0.4, i=1,2,(3)$ and $\Delta \phi\left(j_{i}, p_{T}^{\text {miss }}\right)>0.2, i>3$ are required to remove the events with the large $E_{T}^{\text {miss }}$ from the mis-measurement of the jet energy.

- In order to cover different kinematical regions, we define six signal regions according to $p_{T}\left(j_{1}\right), m_{\text {eff }}($ inclu $)$ and $E_{T}^{\text {miss }} / \sqrt{H_{T}}$ in Table 1 .

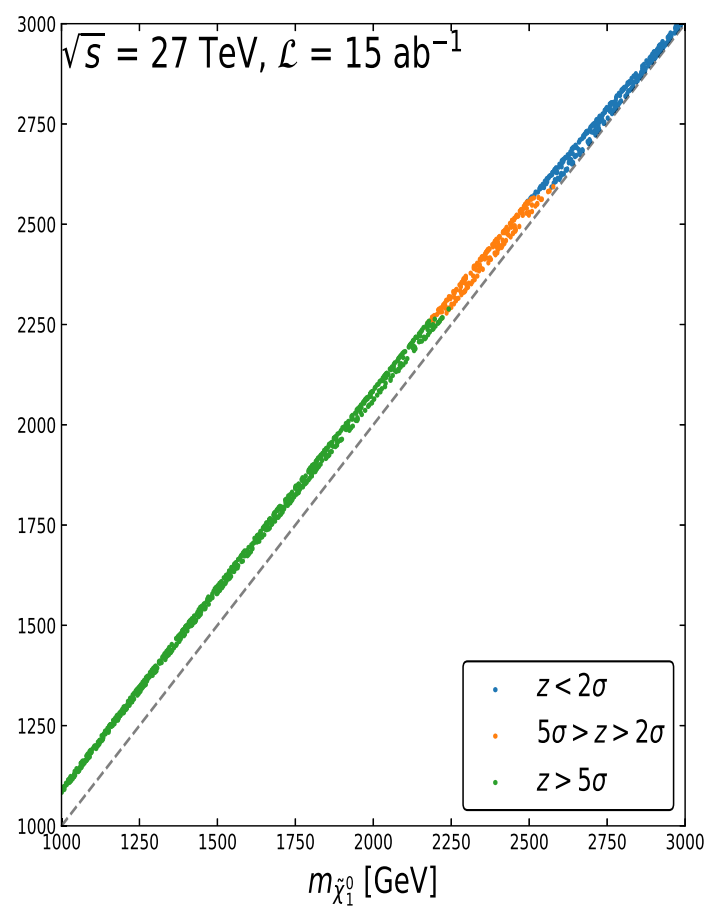

and $15 \mathrm{ab}^{-1}$. The projected samples are those satisfying the constraints in Fig. 1. The green, orange and blue bullets correspond to $Z>5 \sigma$, $2 \sigma<Z<5 \sigma$ and $Z<2 \sigma$, respectively

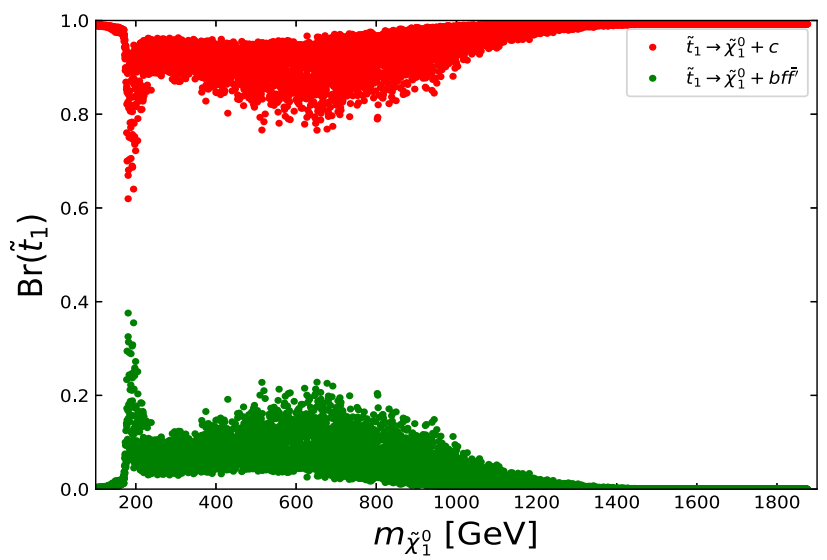

Fig. 6 The branching ratio of $\tilde{t}_{1}$ in the stop coannihilation

\subsection{Stop coannihilation}

The dominant stop pair production processes are $g g \rightarrow \tilde{t} \tilde{t}^{*}$ and $q \bar{q} \rightarrow \tilde{t} \tilde{t}^{*}$ at the HE-LHC. In the stop coannihilation, 


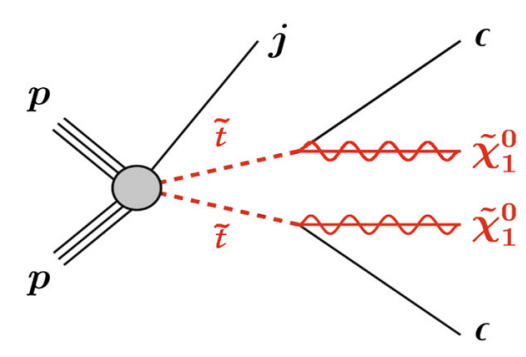

Fig. 7 The schematic diagram of the production process $p p \rightarrow \tilde{t}_{1} \tilde{t}_{1}^{*} j$ with the sequent decay $\tilde{t}_{1} \rightarrow c \tilde{\chi}_{1}^{0}$ at the HE-LHC

From Fig. 6, we can see that the stop mainly decays through the loop-induced flavor changing neutral current process $\tilde{t} \rightarrow$ $c \tilde{\chi}_{1}^{0}$, which is followed by the three-body decay channel $\tilde{t}_{1} \rightarrow$ bf $\bar{f}^{\prime} \tilde{\chi}_{1}^{0}$. Besides, the stop co-annihilation requires the stop mass to be close to the LSP neutralino mass so that the light jet from the stop decay is usually too soft to be detected. Thanks to the initial state radiation (ISR) jets, we can boost the stop-pair system to produce the large $E_{T}^{\text {miss }}$ to trigger events, and then suppress backgrounds. Therefore, we utilize the monojet events from the process $p p \rightarrow \tilde{t} \tilde{t}^{*} j+X$ to probe the stop coannihilation at the HE-LHC, as shown in Fig. 7. The dominant SM backgrounds come from $Z(\rightarrow v \bar{v})+$ jets, $W(\rightarrow \ell v)+$ jets $(\ell=e, \mu, \tau)$ and $t \bar{t}$ events.

In Fig. 8, we present the normalized distributions of the leading jet $p_{T}\left(j_{1}\right)$ and the $E_{T}^{\text {miss }}$ for the signal and backgrounds. It can be seen that the signal has much harder leading jet than the backgrounds. Since the hardness of the event is determined by the $p_{T}$ of the leading jet, the slopes of the leading jet $p_{T}$ distribution in high $p_{T}$ region are almost the same for different stop mass. The $E_{T}^{\text {miss }}$ distribution of the signal events has the slowest fall-off. We expect that a hard cut on $E_{T}^{\text {miss }}$ will remove the backgrounds effectively. Therefore, we impose the following event selection criteria:

- Events are selected with a leading jet with $p_{T}\left(j_{1}\right)>$ $200 \mathrm{GeV}$ and $|\eta|<2.4$.

- Events having muons with $p_{T}>10 \mathrm{GeV}$ or electrons with $p_{T}>20 \mathrm{GeV}$ in the final states are vetoed.

- At most four jets with $p_{T}>30 \mathrm{GeV}$ and $|\eta|<2.8$ are allowed.

- The azimuthal angle between the leading jet and missing transverse momentum $\Delta \phi\left(j_{1}, p_{T}^{\text {miss }}\right)>0.4$ is required to remove the events with the large $E_{T}^{\text {miss }}$ from the mismeasurement of the jet energy.

- Five signal regions, $E_{T}^{\text {miss }}>200,400,600,800,1000$ $\mathrm{GeV}$, are defined in our analysis.

In Fig. 9, we project the surviving samples for stop coannihilation on the plane of $m_{\tilde{t}_{1}}$ versus $m_{\tilde{\chi}_{1}^{0}}$ at the HE-LHC. It can be seen that the HE-LHC is able to probe the DM with a mass $m_{\tilde{\chi}_{1}^{0}}<800$ and $1400 \mathrm{GeV}$ at $Z \geq 5 \sigma$ level for the luminosity $\mathcal{L}=300 \mathrm{fb}^{-1}$ and $15 \mathrm{ab}^{-1}$, respectively. On the other hand, if there was no significant excess, we point out that the DM mass $m_{\tilde{\chi}_{1}^{0}}$ will be excluded up to $1.1 \mathrm{TeV}$ and $1.7 \mathrm{TeV}$ at $Z=2 \sigma$ level, respectively.
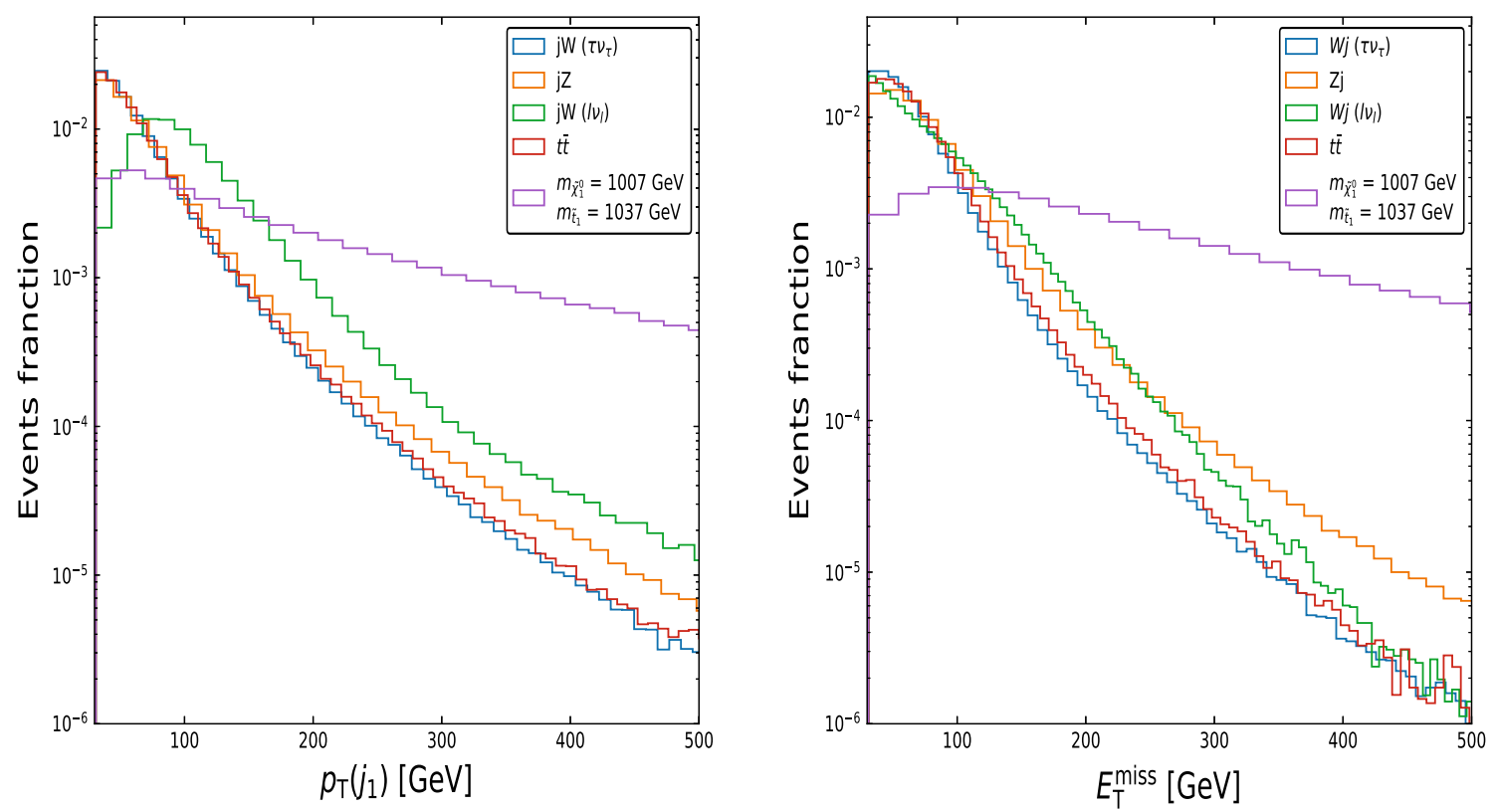

Fig. 8 The normalized distributions of the leading jet $p_{T}\left(j_{1}\right)$ and $E_{T}^{m i s s}$ of the signal and background events at the HE-LHC. The benchmark point is $m_{\tilde{t}_{1}}=1037 \mathrm{GeV}$ and $m_{\tilde{\chi}_{1}^{0}}=1007 \mathrm{GeV}$ 

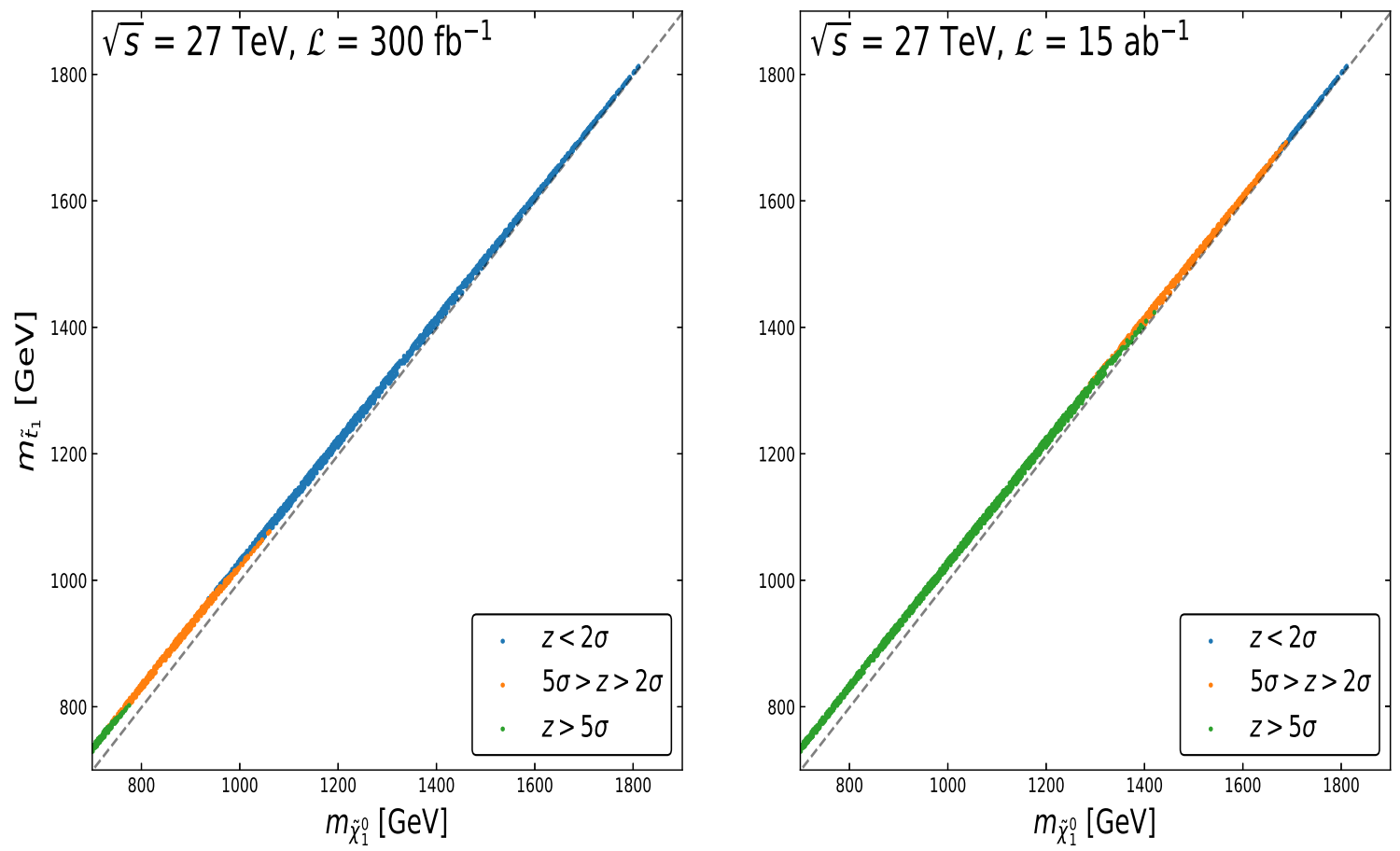

Fig. 9 Same as Fig. 5, but for the monojet events from the process $p p \rightarrow \tilde{t}_{1} \tilde{t}_{1}^{*} j \rightarrow E_{T}^{m i s s}+$ jets

\subsection{Wino coannihilation}

The wino-like electroweakinos $\tilde{\chi}_{1}^{ \pm}$and $\tilde{\chi}_{2}^{0}$ can be produced through the Drell-Yan process $p p \rightarrow \tilde{\chi}_{1}^{ \pm} \tilde{\chi}_{2}^{0}$ at the HE-LHC. From Fig. 10, we can see that the next-to-lightest neutralino $\tilde{\chi}_{2}^{0}$ will dominantly decay via the three-body process $\tilde{\chi}_{2}^{0} \rightarrow$ $\tilde{\chi}_{1}^{0} Z^{*} \rightarrow \tilde{\chi}_{1}^{0} f \bar{f}$. Subsequently, the loop process $\tilde{\chi}_{2}^{0} \rightarrow \gamma \tilde{\chi}_{1}^{0}$ can have the branching ratio of $10 \% \div 30 \%$, which may provide a distinctive signature of a soft photon plus large missing transverse energy with a hard ISR jet at the LHC [86]. The lightest chargino $\tilde{\chi}_{1}^{ \pm}$will proceed through the threebody decay process $\tilde{\chi}_{1}^{ \pm} \rightarrow \tilde{\chi}_{1}^{0} W^{ \pm *} \rightarrow \tilde{\chi}_{1}^{0} f \bar{f}^{\prime}$. Given the compressed spectrum of wino coannihilation, we perform the Monte Carlo simulation of the process $p p \rightarrow \tilde{\chi}_{1}^{ \pm} \tilde{\chi}_{2}^{0} \rightarrow$ $\ell^{+} \ell^{-}+E_{T}^{\text {miss }}+$ jets at the HE-LHC, as shown in Fig. 11. The dominant SM backgrounds in this scenario are Drell-Yan process $p p \rightarrow \gamma^{*} / Z^{*}+$ jets, the dibosons $(W W, W Z, Z Z$ ) and the single top. In contrast with the conventional monojet analysis for the compressed electroweakinos [87], there is no upper limit on the number of jets in our analysis. Instead, we require a small transverse mass $m_{T}\left(\ell, \nu_{\ell}\right)$ to suppress the $t \bar{t}$ background. Besides, the soft lepton pair from the decay of $\tilde{\chi}_{2}^{0}$ can be used as a handle to reduce the huge background $V+$ jets.

In Fig. 12, we show the normalized distribution of the dilepton invariant mass $m_{\ell \ell}$ and the ratio of $E_{T}^{\text {miss }} / H_{T}^{\text {lep }}$ of the signal and background events. We can find that the signal has more events in the range of the invariant mass $m_{\ell \ell} \leq$ $30 \mathrm{GeV}[88,89]$, which can highly suppress the $\gamma^{*} / Z^{(*)}(\rightarrow$

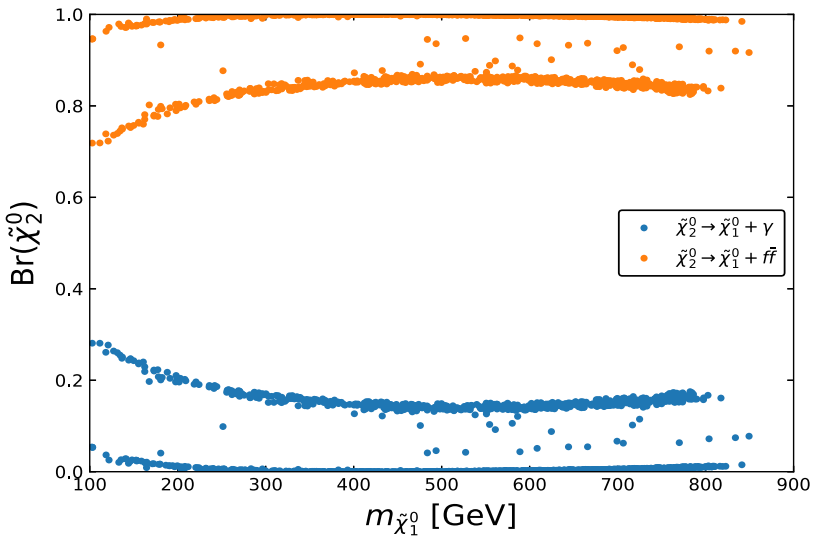

Fig. 10 The branching ratio of $\tilde{\chi}_{1}^{ \pm}$and $\tilde{\chi}_{2}^{0}$ in the wino coannihilation

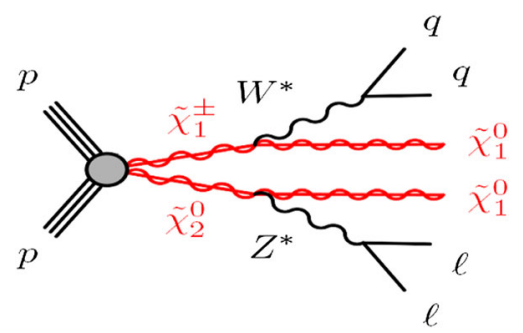

Fig. 11 The schematic diagram of the production process $p p \rightarrow$ $\tilde{\chi}_{1}^{ \pm} \tilde{\chi}_{2}^{0}$ with the sequent decays $\tilde{\chi}_{2}^{0} \rightarrow Z\left(\rightarrow \ell^{+} \ell^{-}\right) \tilde{\chi}_{1}^{0}$ and $\tilde{\chi}_{1}^{ \pm} \rightarrow$ $W(\rightarrow q \bar{q}) \tilde{\chi}_{1}^{0}$ 

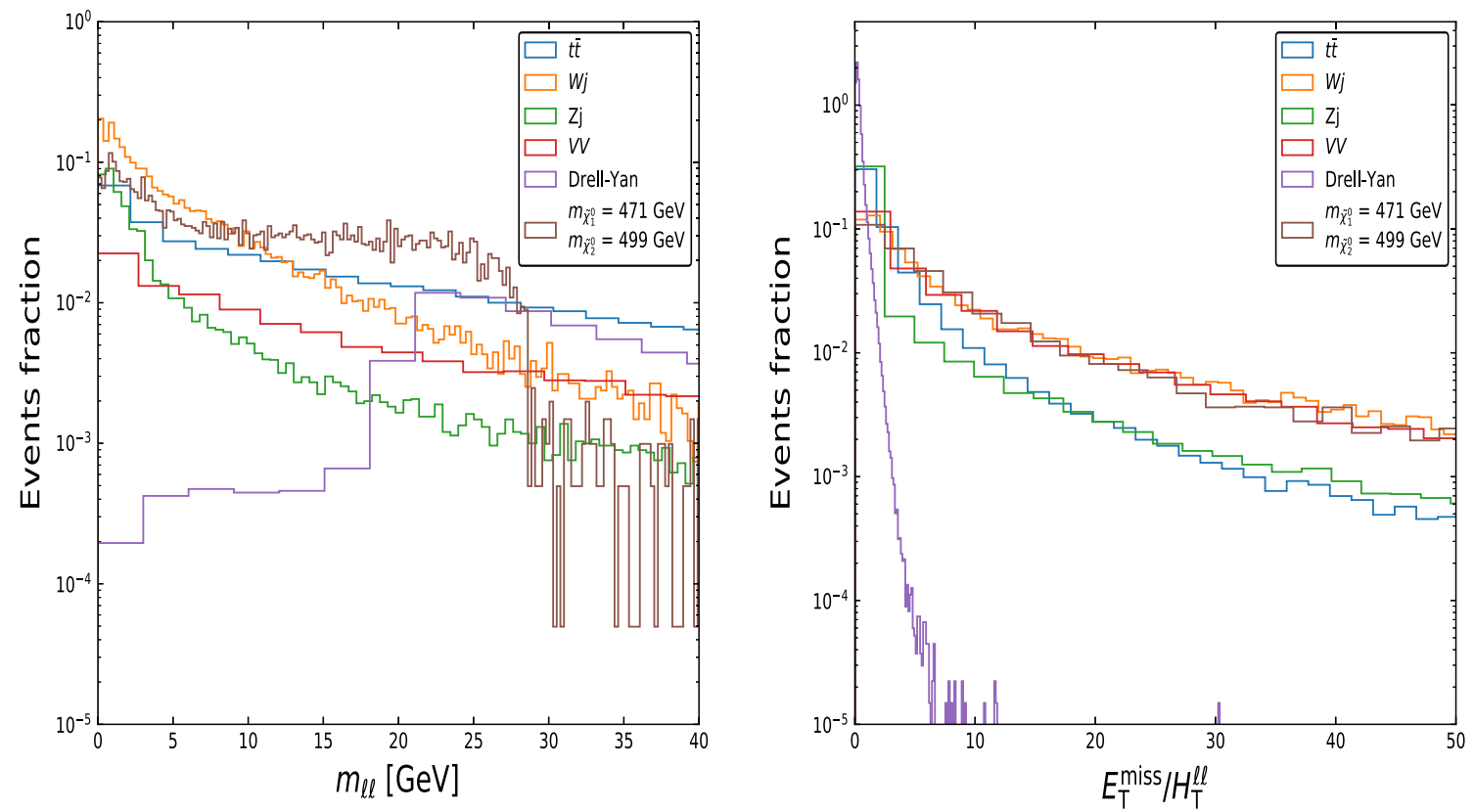

Fig. 12 The normalized distributions of $m_{\ell \ell}$ and $E_{T}^{\text {miss }} / H_{T}^{\text {lep }}$ of the signal and background events at the HE-LHC. The benchmark point is $m_{\tilde{\chi}_{2}^{0}}=499 \mathrm{GeV}, m_{\tilde{\chi}_{1}^{ \pm}}=498 \mathrm{GeV}$ and $m_{\tilde{\chi}_{1}^{0}}=471 \mathrm{GeV}$

Table 2 Ten signal regions are defined by the values of $m_{\ell \ell}$ and $E_{T}^{m i s s} / H_{T}^{l e p}$

\begin{tabular}{llllll}
\hline SRs & SR1 & SR2 & SR3 & SR4 & SR5 \\
\hline$m_{\ell_{1} \ell_{2}}(\mathrm{GeV}) \in$ & {$[5,10]$} & {$[10,15]$} & {$[15,20]$} & {$[20,25]$} & 6 \\
$E_{\mathrm{T}}^{\text {miss }} / H_{\mathrm{T}}^{\text {lep }}>$ & 12 & 10 & 8 & {$[25,30]$} \\
\hline SRs & SR6 & SR7 & SR8 & SR9 & SR10 \\
\hline$m_{\ell_{1} \ell_{2}}(\mathrm{GeV}) \in$ & {$[1,3] \cup[5,30]$} & {$[5,30]$} & {$[10,30]$} & {$[15,30]$} & 8 \\
$E_{\mathrm{T}}^{\text {miss }} / H_{\mathrm{T}}^{\text {lep }}>$ & 14 & 12 & 10 & $620,30]$ \\
\hline
\end{tabular}

$\ell \ell)+j e t s$ and $t \bar{t}$ backgrounds. Besides, it can also hurt the fake/non-prompt leptons effectively [90]. On the other hand, the signal events of the compressed electroweakinos production predict a small value of the scalar sum of the lepton transverse momenta $H_{T}^{l e p}=p_{T}^{\ell_{1}}+p_{T}^{\ell_{2}}$. Thus, the ratio $E_{T}^{\text {miss }} / H_{T}^{\text {lep }}$ can significantly reduce the Drell-Yan and QCD multijet backgrounds [91].

According to the features of above distributions, we perform the following kinematical cuts.

- Events are required to have exactly two same flavor opposite sign leptons $\left(e^{+} e^{-}\right.$or $\left.\mu^{+} \mu^{-}\right)$. The leading and subleading leptons should have transverse momentum larger than 5 and $4 \mathrm{GeV}$, respectively. The separation $\Delta R=\sqrt{\Delta \eta^{2}+\Delta \phi^{2}}$ between two leptons are between 0.05 and 2 .

- We select events with $E_{T}^{\text {miss }}>200 \mathrm{GeV}$ and require the transverse momentum of the leading jet $p_{T}\left(j_{1}\right)>100$
$\mathrm{GeV}$ and $\Delta \phi\left(j_{1}, E_{T}^{m i s s}\right)>2$. We veto any events with $b$-jets.

- To reduce the mismeasurement effect, the events with the azimuthal angle between any jet and $E_{T}^{m i s s}$ smaller than 0.35 is discarded.

- We require the transverse mass $m_{T}\left(\ell_{1}, E_{T}^{m i s s}\right)$ of the leading lepton to be less than $60 \mathrm{GeV}$, which can reduce $t \bar{t}$ significantly.

- We define ten signal regions by the values of $m_{\ell \ell}$ and $E_{T}^{m i s s} / H_{T}^{\text {lep }}$, as shown Table 2.

In Fig. 13, we present the signal significance of the soft lepton pair plus missing transverse energy events from the process $p p \rightarrow \tilde{\chi}_{1}^{ \pm} \tilde{\chi}_{2}^{0}+X$ at the HE-LHC. Compared with the Fig. 1, we notice that the $2 \sigma$ exclusion limit of the neutralino DM mass will be extended from about $180 \mathrm{GeV}$ at the current LHC to $560 \mathrm{GeV}$ at the HE-LHC with the luminosity $\mathcal{L}=$ $300 \mathrm{fb}^{-1}$. Besides, the neutralino DM with the mass less than about $470 \mathrm{GeV}$ can be probed at $Z \geq 5 \sigma$ level at the $\mathrm{HE}$ - 

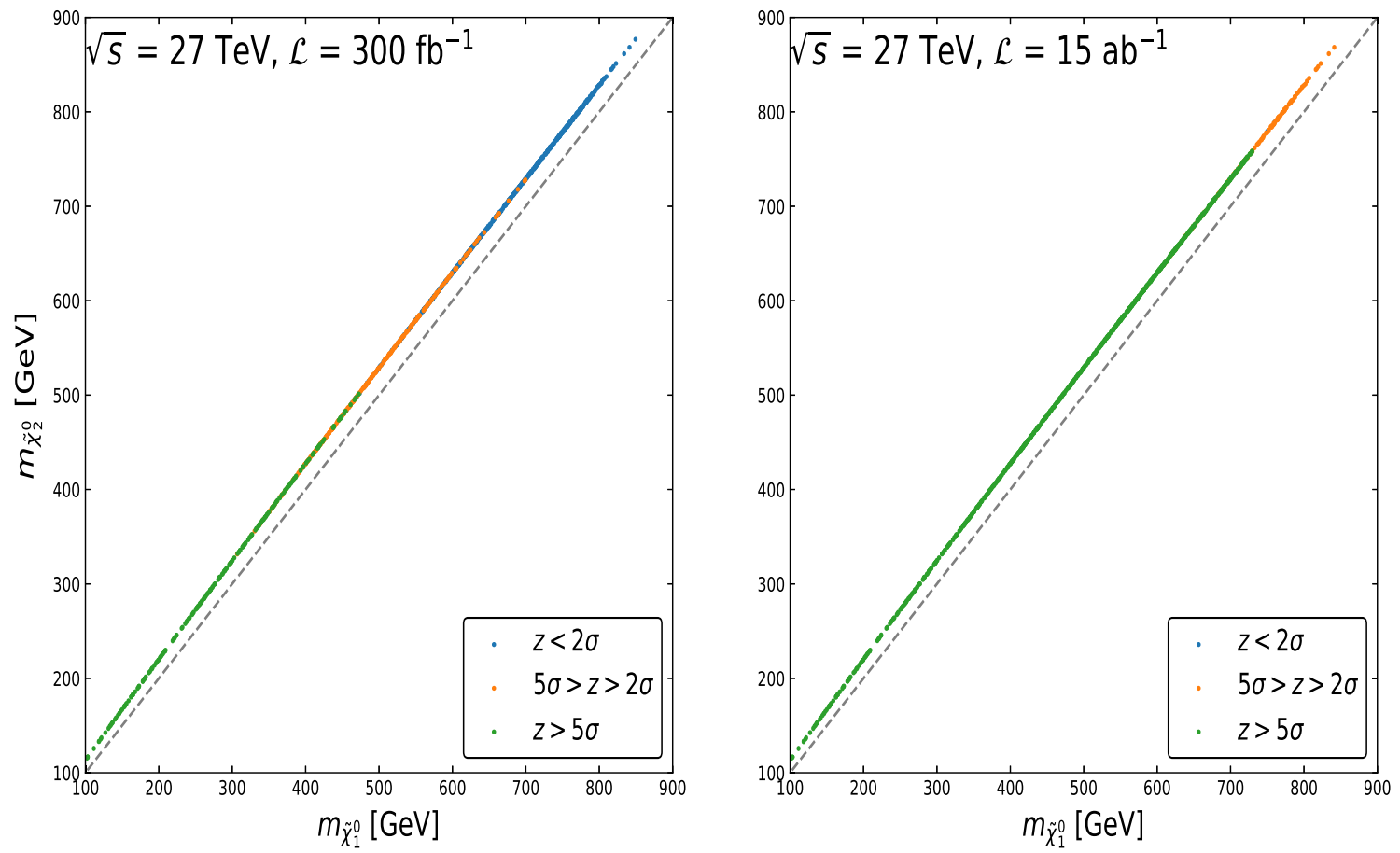

Fig. 13 Same as Fig. 5, but for the soft lepton pair plus missing transverse energy events from the process $p p \rightarrow \tilde{\chi}_{1}^{ \pm} \tilde{\chi}_{2}^{0} \rightarrow \ell^{+} \ell^{-}+E_{T}^{m i s s}+$ jets

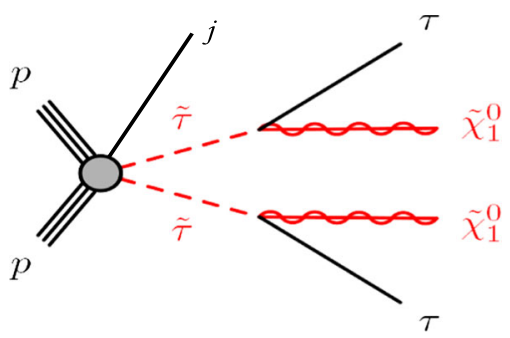

Fig. 14 The schematic diagram of the production process $p p \rightarrow$ $\tilde{\tau} \tilde{\tau}^{*} j+X$ with the sequent decays $\tilde{\tau} \rightarrow \tau \tilde{\chi}_{1}^{0}$

LHC, which will be improved up to about $620 \mathrm{GeV}$ as the luminosity increasing to $15 \mathrm{ab}^{-1}$.

\subsection{Stau coannihilation}

The search for a light stau is experimentally difficult due to its extremely low production rate at the LHC. The staus can be produced directly in pairs through the channel $p p \rightarrow \tilde{\tau} \tilde{\tau}^{*}$. Then, the stau decays with a branching fraction of $100 \%$ to the SM tau-lepton and the LSP neutralino. This will give the signature of $\tau^{+} \tau^{-}+E_{T}^{\text {miss }}$ at the HE-LHC. The hadronic decay of $\tau$ lepton has the largest branching fraction and thus final states with a $\tau_{h}$ provide the best experimental sensitivity. Signal events would thus be characterised by the presence of two sets of close-by hadrons and large $E_{T}^{\text {miss }}$ originating from the invisible LSP and neutrinos. Events are further categorized into regions with different $E_{T}^{\text {miss }}$, to examine different stau mass scenarios. During LHC Run-1, only a narrow parameter region around a stau mass of $109 \mathrm{GeV}$ and a massless lightest neutralino could be excluded by the LHC experiments. Such a mass limit has been extended to $300 \div 400 \mathrm{GeV}$ in the ATLAS Run-2 search of stau [92].

In the stau coannihilation, the small mass difference between stau and LSP neutralino results in low $p_{T}$ visible decay products, making it difficult to identify $\tau$ lepton. Besides, the semi-leptonic decays of $\tau$ lepton leads to lower average $p_{T}$ than hadronic decays, while also being largely indistinguishable from prompt production of electrons and muons. Therefore, we will study the events with one soft hadronically decaying tau lepton and missing transverse energy recoiling against a hard $p_{T}$ jet from ISR, as shown in Fig. 14. The dominant SM backgrounds include the Drell-Yan+jets, $V+$ jets, $t \bar{t}$ and the dibosons $(W W, W Z$, $Z Z$ ).

In Fig. 15, we show the normalized kinematical distributions of the signal and background events. It can be seen that the transverse momentum of the leading jet $p_{T}\left(j_{1}\right)$ in the signal are much harder than those in the Drell-Yan and $V+$ jets backgrounds. On the other hand, the transverse momentum of the hadronic tau $p_{T}\left(\tau_{h}\right)$ from the decay of stau are very soft, most of which distribute in the region of $p_{T}\left(\tau_{h}\right)<35 \mathrm{GeV}$. In addition, the signal has a larger missing transverse energy $E_{T}^{m i s s}$ than all backgrounds. Another sensitive discriminator is the transverse mass $m_{T_{\tau_{h}}}$ between $\tau_{h}$ and $p_{T}^{\text {miss }}$, which is defined as 

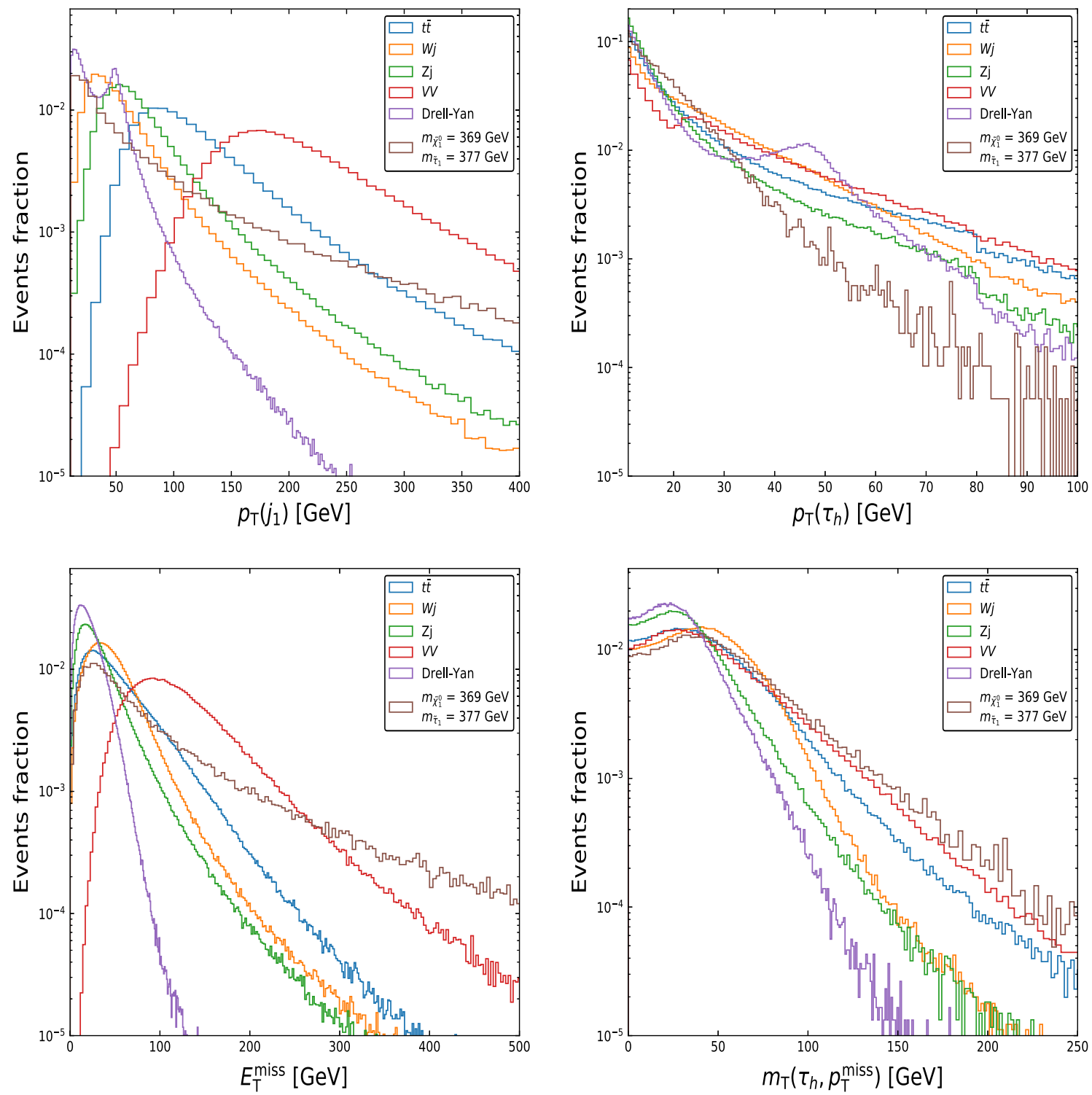

Fig. 15 The normalized distributions of $p_{T}\left(j_{1}\right), p_{T}\left(\tau_{h}\right), E_{T}^{m i s s}$ and $m_{T_{\tau_{h}}}$ of the signal and backgrounds at 27 TeV HE-LHC. The benchmark point is $m_{\tilde{\chi}_{1}^{0}}=369 \mathrm{GeV}, m_{\tilde{\tau}_{1}}=377 \mathrm{GeV}$

Table 3 Three signal regions are defined by the values of $m_{T_{\tau_{h}}}$

\begin{tabular}{llll}
\hline SRs & SR1 & SR2 & SR3 \\
\hline$m_{T_{\tau_{h}}}(\mathrm{GeV})$ & $>150$ & $>200$ & $>250$ \\
\hline
\end{tabular}

$m_{T_{\tau_{h}}}=\sqrt{2 p_{T}^{m i s s} p_{T}\left(\tau_{h}\right)\left(1-\cos \Delta \phi\left(\tau_{h}, p_{T}^{m i s s}\right)\right)}$.

As comparison with the background, the signal has more events in the range of high values of $m_{T_{\tau_{h}}}$ than the backgrounds. For example, when $m_{T_{\tau_{h}}}>150 \mathrm{GeV}$, almost events of the Drell-Yan process will be removed.

In the selection of the signal events, we impose the following criteria to suppress the backgrounds:
- Events containing any isolated electron or muon, with $p_{T}>20 \mathrm{GeV}$, are vetoed.

- Events with any $b$-jet are rejected. The leading jet $p_{T}\left(j_{1}\right)$ has to be larger than $100 \mathrm{GeV}$.

- Events are required to have exactly one $\tau_{h}$ with $15<$ $p_{T}\left(\tau_{h}\right)<35 \mathrm{GeV}$ and $\left|\eta\left(\tau_{h}\right)\right|<2.3$. The efficiency of tau tagging is assumed as $60 \%$.

- Jets and $\tau_{h}$ are required to be well separated by a cut of $\Delta R\left(\tau_{h}\right.$, jet $)>0.4$, which will reject jets from QCD processes that can mimic the signature of a $\tau_{h}$.

- Events with the missing transverse energy $E_{T}^{\text {miss }}$ larger than $230 \mathrm{GeV}$ are required. Then, the multijets events will becomes negligible.

- We define three signal regions according to the values of $m_{T_{\tau_{h}}}$, which is shown in the Table 3 . 

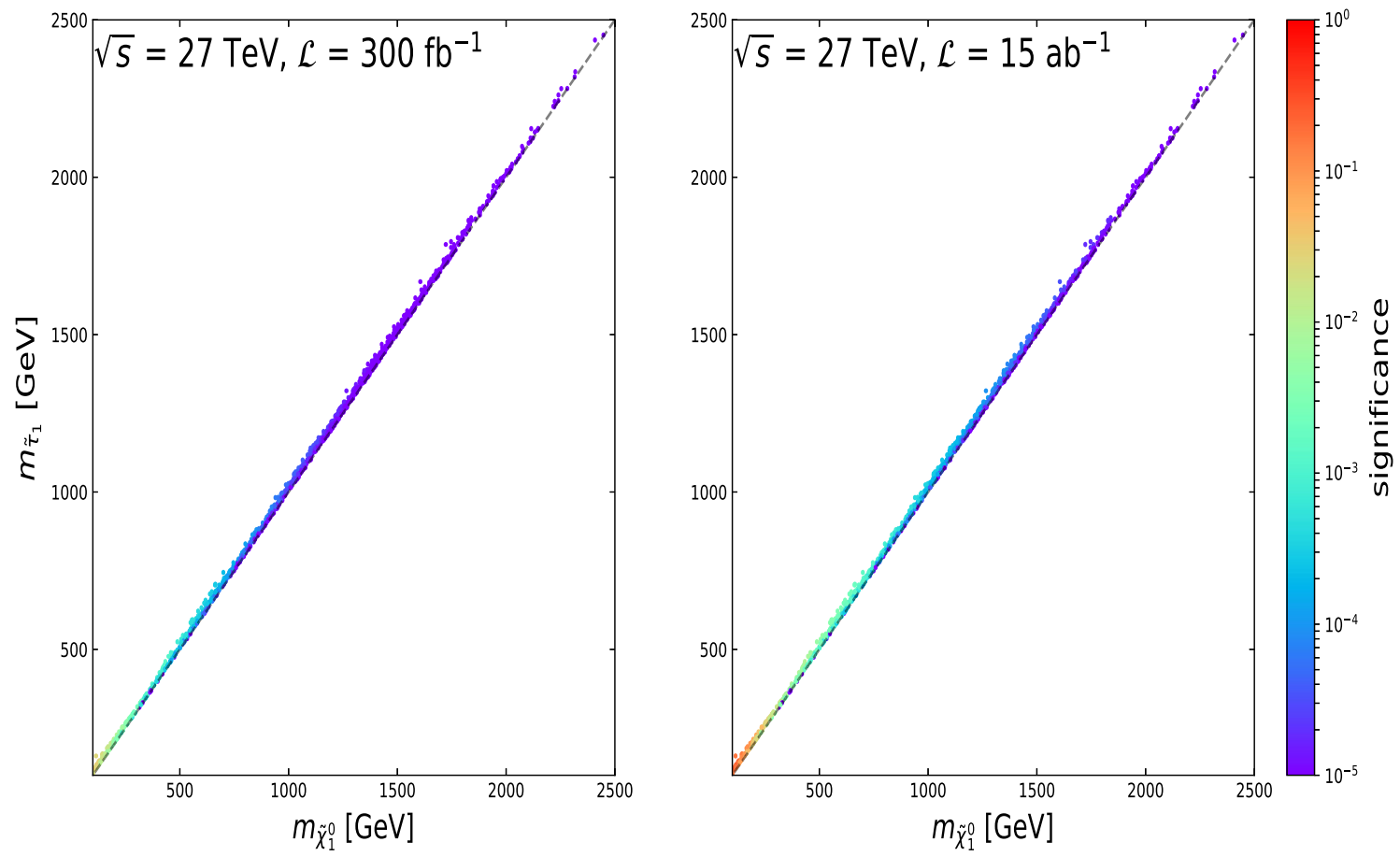

Fig. 16 The statistical significance of the process $p p \rightarrow j \tilde{\tau}_{1} \tilde{\tau}_{1}^{*} \rightarrow j+\tau_{h}+E_{T}^{m i s s}$ at the HE-LHC

Figure 16 shows the statistical significance of the process $p p \rightarrow j \tilde{\tau}_{1} \tilde{\tau}_{1}^{*} \rightarrow j+\tau_{h}+E_{T}^{m i s s}$ at the HE-LHC. We find the significance of all samples in the stau coannihilation is less than $2 \sigma$. In contrast with the above search for the compressed winos, there are two main reasons for such a poor sensitivity: one is that the cross section of stau pair production is relative small, which is about $1 / 4$ of the cross section of wino pair production for the same mass. The other is that tau tagging efficiency for the soft tau from stau decay is badly reduced. We also used the proposed analysis with two tagging hadronic tau lepton [29] and found that it reach a similar sensitivity as ours. Besides, the vector boson fusion topologies with taus in the final states have been proposed [93], however, which has a smaller cross section of the process $p p \rightarrow j j \tilde{\tau} \tilde{\tau}^{*}$ at the HE-LHC.

In above discussions, we present the mass reaches of the gluino, stop, wino and stau in the coannihilations at the HELHC. It should be mentioned that the statistical significance will get degraded when systematic uncertainties are taken into account. The determination of the systematic uncertainties due to the high pile-up conditions of the future hadroncollider runs is beyond the scope of this paper. It must be revisited with the real performance of the upgraded ATLAS and CMS detectors. Besides, the machine learning methods have been recently proposed to enhance the sensitivity in the search of sparticles at the LHC [94-98]. We expect that our results may be improved by using those advanced analysis approaches at the HE-LHC.

\section{Conclusions}

In this paper, we investigate the potential of the discovery of the neutralino DM in the gluino, stop, wino, stau coannihilations at the HE-LHC. We carried out our study in the simplified MSSM model that only includes the relevant sparticles in each scenario. We firstly impose the relic density constraint and determine the allowed parameter space of the gluino, stop, wino and stau coannihilations. Since the mass difference between the neutralino DM and its coannihilating partner is usually small, the discovery of coannihilating partner will also provide a measurement of the neutralino DM mass. Thus, we perform the Monte Carlo simulations to investigate the observability of gluino, stop, wino and stau in each coannihilation scenario at the HE-LHC. Our analysis strategies include the multijet with $E_{T}^{\text {miss }}$, the monojet, the soft lepton pair plus $E_{T}^{m i s s}$, and the monojet plus a hadronic tau. In this end, it is found that the neutralino DM mass can be excluded up to $2.6,1.7$ and $0.8 \mathrm{TeV}$ through the processes $p p \rightarrow \tilde{g} \tilde{g} \rightarrow j e t s+E_{T}^{m i s s}, p p \rightarrow \tilde{t} \tilde{t}^{*} j \rightarrow j+E_{T}^{m i s s}$ and $p p \rightarrow \tilde{\chi}_{1}^{ \pm} \tilde{\chi}_{2}^{0} \rightarrow \ell^{+} \ell^{-}+E_{T}^{\text {miss }}+$ jets for the gluino, stop and wino coannihilations at the $2 \sigma$ level, respectively. While there is still no sensitivity of the neutralino DM through the process $p p \rightarrow j \tilde{\tau}_{1} \tilde{\tau}_{1}^{*} \rightarrow j+\tau_{h}+E_{T}^{\text {miss }}$ in stau coannihilation at the HE-LHC, because of the low rate of the direct stau pair production and the soft tau from the decay of stau.

Acknowledgements Part of this work was done while M. A. was visiting Nanjing Normal University. This work was supported by the 
National Natural Science Foundation of China (NNSFC) under Grant nos. 11705093 and 11675242 .

Data Availability Statement This manuscript has no associated data or the data will not be deposited. [Authors' comment: This is a theoretical study and no experimental data.]

Open Access This article is licensed under a Creative Commons Attribution 4.0 International License, which permits use, sharing, adaptation, distribution and reproduction in any medium or format, as long as you give appropriate credit to the original author(s) and the source, provide a link to the Creative Commons licence, and indicate if changes were made. The images or other third party material in this article are included in the article's Creative Commons licence, unless indicated otherwise in a credit line to the material. If material is not included in the article's Creative Commons licence and your intended use is not permitted by statutory regulation or exceeds the permitted use, you will need to obtain permission directly from the copyright holder. To view a copy of this licence, visit http://creativecomm ons.org/licenses/by/4.0/.

Funded by SCOAP 3 .

\section{References}

1. B.W. Lee, S. Weinberg, Cosmological lower bound on heavy neutrino masses. Phys. Rev. Lett. 39, 165 (1977). https://doi.org/10. 1103/PhysRevLett.39.165

2. K. Griest, M. Kamionkowski, Unitarity limits on the mass and radius of dark matter particles. Phys. Rev. Lett. 64, 615 (1990). https://doi.org/10.1103/PhysRevLett.64.615

3. G. Arcadi, M. Dutra, P. Ghosh, M. Lindner, Y. Mambrini, M. Pierre et al., The waning of the WIMP? A review of models, searches, and constraints. Eur. Phys. J. C 78, 203 (2018). https://doi.org/10.1140/ epjc/s10052-018-5662-y. [arXiv:1703.07364]

4. N. Arkani-Hamed, A. Delgado, G.F. Giudice, The well-tempered neutralino. Nucl. Phys. B 741, 108 (2006). https://doi.org/10.1016/ j.nuclphysb.2006.02.010. arXiv:hep-ph/0601041

5. K. Griest, D. Seckel, Three exceptions in the calculation of relic abundances. Phys. Rev. D 43, 3191 (1991). https://doi.org/10.1103/ PhysRevD.43.3191

6. J.R. Ellis, T. Falk, K.A. Olive, Neutralino-stau coannihilation and the cosmological upper limit on the mass of the lightest supersymmetric particle. Phys. Lett. B 444, 367 (1998). https://doi.org/10. 1016/S0370-2693(98)01392-6. arXiv:hep-ph/9810360

7. J.R. Ellis, T. Falk, K.A. Olive, M. Srednicki, Calculations of neutralino-stau coannihilation channels and the cosmologically relevant region of MSSM parameter space. Astropart. Phys. 13, 181 (2000). https://doi.org/10.1016/S0927-6505(99)00104-8. arXiv:hep-ph/9905481

8. J.R. Ellis, K.A. Olive, Y. Santoso, Calculations of neutralino stop coannihilation in the CMSSM. Astropart. Phys. 18, 395 (2003). https://doi.org/10.1016/S0927-6505(02)00151-2. arXiv:hep-ph/0112113

9. J. Ellis, K.A. Olive, J. Zheng, The extent of the stop coannihilation strip. Eur. Phys. J. C 74, 2947 (2014). https://doi.org/10.1140/epjc/ s10052-014-2947-7. arXiv:1404.5571

10. N. Desai, J. Ellis, F. Luo, J. Marrouche, Closing in on the tip of the CMSSM stau coannihilation strip. Phys. Rev. D 90, 055031 (2014). https://doi.org/10.1103/PhysRevD.90.055031. arXiv:1404.5061

11. M. Citron, J. Ellis, F. Luo, J. Marrouche, K.A. Olive, K.J. de Vries, End of the CMSSM coannihilation strip is nigh. Phys. Rev. D 87, 036012 (2013). https://doi.org/10.1103/PhysRevD.87.036012. arXiv: 1212.2886

12. C. Han, K.-I. Hikasa, L. Wu, J.M. Yang, Y. Zhang, Status of CMSSM in light of current LHC Run-2 and LUX data. Phys.
Lett. B 769, 470 (2017). https://doi.org/10.1016/j.physletb.2017. 04.026. arXiv: 1612.02296

13. A. Birkedal-Hansen, E.-H. Jeong, Gaugino and Higgsino coannihilations. 1. Neutralino neutralino interactions. JHEP 02, 047 (2003). https://doi.org/10.1088/1126-6708/2003/02/047. arXiv:hep-ph/0210041

14. S. Profumo, C.E. Yaguna, Gluino coannihilations and heavy bino dark matter. Phys. Rev. D 69, 115009 (2004). https://doi.org/10. 1103/PhysRevD.69.115009. arXiv:hep-ph/0402208

15. H. Baer, T. Krupovnickas, A. Mustafayev, E.-K. Park, S. Profumo, $\mathrm{X}$. Tata, Exploring the BWCA (bino-wino co-annihilation) scenario for neutralino dark matter. JHEP 12, 011 (2005). https://doi.org/ 10.1088/1126-6708/2005/12/011. arXiv:hep-ph/0511034

16. D. Feldman, Z. Liu, P. Nath, Gluino NLSP, dark matter via gluino coannihilation, and LHC signatures. Phys. Rev. D 80, 015007 (2009). https://doi.org/10.1103/PhysRevD.80.015007. arXiv:0905.1148

17. M. Ibe, A. Kamada, S. Matsumoto, Mixed (cold+warm) dark matter in the bino-wino coannihilation scenario. Phys. Rev. D 89, 123506 (2014). https://doi.org/10.1103/PhysRevD.89.123506. arXiv: 1311.2162

18. K. Harigaya, K. Kaneta, S. Matsumoto, Gaugino coannihilations. Phys. Rev. D 89, 115021 (2014). https://doi.org/10.1103/ PhysRevD.89.115021. arXiv:1403.0715

19. J. Ellis, J.L. Evans, F. Luo, K.A. Olive, Scenarios for gluino coannihilation. JHEP 02, 071 (2016). https://doi.org/10.1007/ JHEP02(2016)071. arXiv: 1510.03498

20. J. Ellis, F. Luo, K.A. Olive, Gluino coannihilation revisited. JHEP 09, 127 (2015). https://doi.org/10.1007/JHEP09(2015)127. arxiv: 1503.07142

21. T.T. Yanagida, W. Yin, N. Yokozaki, Bino-wino coannihilation as a prediction in the $E_{7}$ unification of families. arXiv:1907.07168

22. ATLAS collaboration, Search for electroweak production of supersymmetric states in scenarios with compressed mass spectra at $\sqrt{s}=13 \mathrm{TeV}$ with the ATLAS detector. Phys. Rev. D 97, 052010 (2018). https://doi.org/10.1103/PhysRevD.97.052010. arXiv: 1712.08119

23. ATLAS collaboration, Search for direct pair production of higgsinos by the reinterpretation of the disappearing track analysis with $36.1 \mathrm{fb}^{-1}$ of $\sqrt{s}=13 \mathrm{TeV}$ data collected with the ATLAS experiment, ATL-PHYS-PUB-2017-019. https://cds.cern. ch/record/2297480

24. ATLAS collaboration, ATLAS sensitivity to winos and higgsinos with a highly compressed mass spectrum at the HL-LHC, ATLPHYS-PUB-2018-031. https://cds.cern.ch/record/2647294

25. R.L. Arnowitt, B. Dutta, T. Kamon, N. Kolev, D.A. Toback, Detection of SUSY in the stau-neutralino coannihilation region at the LHC. Phys. Lett. B 639, 46 (2006). https://doi.org/10.1016/j. physletb.2006.07.049, https://doi.org/10.1016/j.physletb.2006.05. 090. arXiv:hep-ph/0603128

26. V. Khotilovich, R.L. Arnowitt, B. Dutta, T. Kamon, The stau neutralino co-annihilation region at an international linear collider. Phys. Lett. B 618, 182 (2005). https://doi.org/10.1016/j.physletb. 2005.04.078. arXiv:hep-ph/0503165

27. M. Berggren, A. Cakir, D. Krücker, J. List, I.A. MelzerPellmann, B. Safarzadeh Samani et al., Non-simplified SUSY: $\tilde{\tau}$-coannihilation at LHC and ILC. Eur. Phys. J. C 76, 183 (2016). https://doi.org/10.1140/epjc/s10052-016-3914-2. arXiv: 1508.04383

28. A. Flórez, L. Bravo, A. Gurrola, C. Ávila, M. Segura, P. Sheldon et al., Probing the stau-neutralino coannihilation region at the LHC with a soft tau lepton and a jet from initial state radiation. Phys. Rev. D 94, 073007 (2016). https://doi.org/10.1103/PhysRevD.94. 073007. arXiv: 1606.08878

29. A. Aboubrahim, P. Nath, A.B. Spisak, Stau coannihilation, compressed spectrum, and SUSY discovery potential at the LHC. Phys. 
Rev. D 95, 115030 (2017). https://doi.org/10.1103/PhysRevD.95. 115030. arXiv: 1704.04669

30. M. Drees, M. Hanussek, J.S. Kim, Light stop searches at the LHC with monojet events. Phys. Rev. D 86, 035024 (2012). https://doi. org/10.1103/PhysRevD.86.035024. arXiv:1201.5714

31. Z.-H. Yu, X.-J. Bi, Q.-S. Yan, P.-F. Yin, Detecting light stop pairs in coannihilation scenarios at the LHC. Phys. Rev. D 87, 055007 (2013). https://doi.org/10.1103/PhysRevD.87.055007. arXiv: 1211.2997

32. A. Kobakhidze, N. Liu, L. Wu, J.M. Yang, M. Zhang, Closing up a light stop window in natural SUSY at LHC. Phys. Lett. B 755, 76 (2016). https://doi.org/10.1016/j.physletb.2016.02.003. arXiv:1511.02371

33. P. Nath, A.B. Spisak, Gluino coannihilation and observability of gluinos at LHC RUN II. Phys. Rev. D 93, 095023 (2016). https:// doi.org/10.1103/PhysRevD.93.095023. arXiv:1603.04854

34. N. Nagata, H. Otono, S. Shirai, Probing bino-cgluino coannihilation at the LHC. Phys. Lett. B 748, 24 (2015). https://doi.org/10. 1016/j.physletb.2015.06.044. arXiv:1504.00504

35. G.H. Duan, K.-I. Hikasa, J. Ren, L. Wu, J.M. Yang, Probing binowino coannihilation dark matter below the neutrino floor at the LHC. Phys. Rev. D 98, 015010 (2018). https://doi.org/10.1103/ PhysRevD.98.015010. arXiv:1804.05238

36. N. Nagata, H. Otono, S. Shirai, Probing bino-wino coannihilation at the LHC. JHEP 10, 086 (2015). https://doi.org/10.1007/ JHEP10(2015)086. arXiv:1506.08206

37. N. Nagata, Probing gaugino coannihilation with displaced vertex searches. PoS DSU2015, 036 (2016). https://doi.org/10.22323/1. 268.0036. arXiv: 1605.00585

38. FCC collaboration, HE-LHC: the high-energy large hadron collider. Eur. Phys. J. ST 228, 1109 (2019). https://doi.org/10.1140/ epjst/e2019-900088-6

39. WORKING GROUP 3 collaboration, Beyond the standard model physics at the HL-LHC and HE-LHC. arXiv:1812.07831

40. ATLAS, CMS collaboration, Report on the physics at the HLLHC and perspectives for the HE-LHC, in HL/HE-LHC Physics Workshop: Final Jamboree Geneva, CERN, March 1, 2019 (2019). arXiv: 1902.10229

41. T. Han, S. Mukhopadhyay, X. Wang, Electroweak dark matter at future hadron colliders. Phys. Rev. D 98, 035026 (2018). https:// doi.org/10.1103/PhysRevD.98.035026. arXiv:1805.00015

42. H. Baer, V. Barger, J.S. Gainer, D. Sengupta, H. Serce, X. Tata, LHC luminosity and energy upgrades confront natural supersymmetry models. Phys. Rev. D 98, 075010 (2018). https://doi.org/10.1103/ PhysRevD.98.075010. arXiv:1808.04844

43. A. Aboubrahim, P. Nath, Naturalness, the hyperbolic branch, and prospects for the observation of charged Higgs bosons at high luminosity LHC and 27 TeV LHC. Phys. Rev. D 98, 095024 (2018). https://doi.org/10.1103/PhysRevD.98.095024. arXiv:1810.12868

44. L. Di Luzio, R. Gröber, G. Panico, Probing new electroweak states via precision measurements at the LHC and future colliders. JHEP 01, 011 (2019). https://doi.org/10.1007/JHEP01(2019)011. arXiv: 1810.10993

45. A. Aboubrahim, P. Nath, Supersymmetry at a $28 \mathrm{TeV}$ hadron collider: HE-LHC. Phys. Rev. D 98, 015009 (2018). https://doi.org/ 10.1103/PhysRevD.98.015009. arXiv: 1804.08642

46. T. Han, A. Ismail, B.Shams Es Haghi, SUSY signals from QCD production at the upgraded LHC. Phys. Lett. B 793, 354 (2019). https://doi.org/10.1016/j.physletb.2019.05.004. arXiv: 1902.05109

47. A. Aboubrahim, P. Nath, Detecting hidden sector dark matter at HL-LHC and HE-LHC via long-lived stau decays. Phys. Rev. D 99, 055037 (2019). https://doi.org/10.1103/PhysRevD.99.055037. arXiv: 1902.05538

48. A. Aboubrahim, P. Nath, Mixed hidden sector-visible sector dark matter and observation of a CP odd Higgs boson at HL-LHC and
HE-LHC. Phys. Rev. D 100, 015042 (2019). https://doi.org/10. 1103/PhysRevD.100.015042. arXiv:1905.04601

49. J.F. Gunion, H.E. Haber, Higgs Bosons in supersymmetric models. 1. Nucl. Phys. B 272, 1 (1986). https:// doi.org/10.1016/0550-3213(86)90340-8, https://doi.org/10.1016/ 0550-3213(93)90653-7

50. M.J. Baker et al., The coannihilation codex. JHEP 12, 120 (2015). https://doi.org/10.1007/JHEP12(2015)120. arXiv:1510.03434

51. G. Belanger, F. Boudjema, A. Pukhov, A. Semenov, micrOMEGAs3: a program for calculating dark matter observables. Comput. Phys. Commun. 185, 960 (2014). https://doi.org/ 10.1016/j.cpc.2013.10.016. arXiv:1305.0237

52. A. Djouadi, M.M. Muhlleitner, M. Spira, Decays of supersymmetric particles: the program SUSY-HIT (SUspect-SdecaYHdecay-InTerface). Acta Phys. Polon. B 38, 635 (2007). arXiv:hep-ph/0609292

53. PLANCK collaboration, Planck 2013 results. XVI. Cosmological parameters. Astron. Astrophys. 571, A16 (2014). https://doi.org/ 10.1051/0004-6361/201321591. arXiv:1303.5076

54. ATLAS, CMS collaboration, Combined measurement of the Higgs Boson mass in pp collisions at $\sqrt{s}=7$ and $8 \mathrm{TeV}$ with the ATLAS and CMS experiments. Phys. Rev. Lett. 114, 191803 (2015). https:// doi.org/10.1103/PhysRevLett.114.191803. arXiv:1503.07589

55. D. Chowdhury, R.M. Godbole, K.A. Mohan, S.K. Vempati, Charge and color breaking constraints in MSSM after the Higgs discovery at LHC. JHEP 02, 110 (2014). https://doi.org/10.1007/ JHEP03(2018)149, https://doi.org/10.1007/JHEP02(2014)110. arXiv: 1310.1932

56. T. Kitahara, T. Yoshinaga, Stau with large mass difference and enhancement of the Higgs to diphoton decay rate in the MSSM. JHEP 05, 035 (2013). https://doi.org/10.1007/JHEP05(2013)035. arXiv: 1303.0461

57. G.H. Duan, C. Han, B. Peng, L. Wu, J.M. Yang, Vacuum stability in stau-neutralino coannihilation in MSSM. Phys. Lett. B 788, 475 (2019). https://doi.org/10.1016/j.physletb.2018.12.001. arXiv: 1809.10061

58. ATLAS collaboration, Search for squarks and gluinos in final states with jets and missing transverse momentum using $36 \mathrm{fb}^{-1}$ of $\sqrt{s}=13 \mathrm{TeV}$ pp collision data with the ATLAS detector. Phys. Rev. D 97, 112001 (2018). https://doi.org/10.1103/PhysRevD.97. 112001. arXiv: 1712.02332

59. ATLAS collaboration, Search for dark matter and other new phenomena in events with an energetic jet and large missing transverse momentum using the ATLAS detector. JHEP 01, 126 (2018). https://doi.org/10.1007/JHEP01(2018)126. arXiv:1711.03301

60. J. Hisano, S. Matsumoto, M.M. Nojiri, O. Saito, Non-perturbative effect on dark matter annihilation and gamma ray signature from galactic center. Phys. Rev. D 71, 063528 (2005). https://doi.org/ 10.1103/PhysRevD.71.063528. arXiv:hep-ph/0412403

61. J. Hisano, S. Matsumoto, M. Nagai, O. Saito, M. Senami, Nonperturbative effect on thermal relic abundance of dark matter. Phys. Lett. B 646, 34 (2007). https://doi.org/10.1016/j.physletb.2007.01. 012. arXiv:hep-ph/0610249

62. A. Hryczuk, R. Iengo, P. Ullio, Relic densities including Sommerfeld enhancements in the MSSM. JHEP 03, 069 (2011). https://doi. org/10.1007/JHEP03(2011)069. arXiv:1010.2172

63. A. Hryczuk, The Sommerfeld enhancement for scalar particles and application to sfermion co-annihilation regions. Phys. Lett. B 699, 271 (2011). https://doi.org/10.1016/j.physletb.2011.04.016. arXiv: 1102.4295

64. M. Beneke, C. Hellmann, P. Ruiz-Femenia, Non-relativistic pair annihilation of nearly mass degenerate neutralinos and charginos I. General framework and S-wave annihilation, JHEP 03, 148 (2013). https://doi.org/10.1007/JHEP10(2013)224, https:// doi.org/10.1007/JHEP03(2013)148. arXiv:1210.7928 
65. C. Hellmann, P. Ruiz-Femenía, Non-relativistic pair annihilation of nearly mass degenerate neutralinos and charginos II. $\mathrm{P}$-wave and next-to-next-to-leading order S-wave coefficients. JHEP 08, 084 (2013). https://doi.org/10.1007/JHEP08(2013)084. arXiv: 1303.0200

66. M. Beneke, C. Hellmann, P. Ruiz-Femenia, Non-relativistic pair annihilation of nearly mass degenerate neutralinos and charginos III. Computation of the Sommerfeld enhancements. JHEP 05, 115 (2015). https://doi.org/10.1007/JHEP05(2015)115. arXiv: 1411.6924

67. M. Beneke, C. Hellmann, P. Ruiz-Femenia, Heavy neutralino relic abundance with Sommerfeld enhancements-a study of pMSSM scenarios. JHEP 03, 162 (2015). https://doi.org/10.1007/ JHEP03(2015)162. arXiv:1411.6930

68. M. Beneke, A. Bharucha, F. Dighera, C. Hellmann, A. Hryczuk, S. Recksiegel et al., Relic density of wino-like dark matter in the MSSM. JHEP 03, 119 (2016). https://doi.org/10.1007/ JHEP03(2016)119. arXiv:1601.04718

69. J. Ellis, J.L. Evans, F. Luo, K.A. Olive, J. Zheng, Stop coannihilation in the CMSSM and SubGUT models. Eur. Phys. J. C 78, 425 (2018). https://doi.org/10.1140/epjc/s10052-018-5831-z. arXiv: 1801.09855

70. W. Shepherd, T.M.P. Tait, G. Zaharijas, Bound states of weakly interacting dark matter. Phys. Rev. D 79, 055022 (2009). https:// doi.org/10.1103/PhysRevD.79.055022. arXiv:0901.2125

71. B. von Harling, K. Petraki, Bound-state formation for thermal relic dark matter and unitarity. JCAP 1412, 033 (2014). https://doi.org/ 10.1088/1475-7516/2014/12/033. arXiv: 1407.7874

72. S.P. Liew, F. Luo, Effects of QCD bound states on dark matter relic abundance. JHEP 02, 091 (2017). https://doi.org/10.1007/ JHEP02(2017)091. arXiv:1611.08133

73. T. Binder, L. Covi, K. Mukaida, Dark matter Sommerfeld-enhanced annihilation and bound-state decay at finite temperature. Phys. Rev. D 98, 115023 (2018). https://doi.org/10.1103/PhysRevD.98. 115023. arXiv: 1808.06472

74. H. Fukuda, F. Luo, S. Shirai, How heavy can neutralino dark matter be? JHEP 04, 107 (2019). https://doi.org/10.1007/ JHEP04(2019)107. arXiv:1812.02066

75. T. Moroi, Y. Sumino, A. Yotsuyanagi, QCD correction to neutralino annihilation process and dark matter density in supersymmetric models. Phys. Rev. D 74, 015016 (2006). https://doi.org/10.1103/ PhysRevD.74.015016. arXiv:hep-ph/0605181

76. B. Herrmann, M. Klasen, SUSY-QCD corrections to dark matter annihilation in the Higgs funnel. Phys. Rev. D 76, 117704 (2007). https://doi.org/10.1103/PhysRevD.76.117704. arXiv:0709.0043

77. A. Hryczuk, R. Iengo, The one-loop and Sommerfeld electroweak corrections to the Wino dark matter annihilation. JHEP 01, 163 (2012). https://doi.org/10.1007/JHEP01(2012)163, https:// doi.org/10.1007/JHEP06(2012)137. arXiv:1111.2916

78. T. Bringmann, A.J. Galea, P. Walia, Leading QCD corrections for indirect dark matter searches: a fresh look. Phys. Rev. D 93, 043529 (2016). https://doi.org/10.1103/PhysRevD.93.043529. arXiv: 1510.02473

79. M. Klasen, K. Kovarik, P. Steppeler, SUSY-QCD corrections for direct detection of neutralino dark matter and correlations with relic density. Phys. Rev. D 94, 095002 (2016). https://doi.org/10.1103/ PhysRevD.94.095002. arXiv:1607.06396

80. J. Alwall, R. Frederix, S. Frixione, V. Hirschi, F. Maltoni, O. Mattelaer et al., The automated computation of tree-level and nextto-leading order differential cross sections, and their matching to parton shower simulations. JHEP 07, 079 (2014). https://doi.org/ 10.1007/JHEP07(2014)079. arXiv:1405.0301

81. T. Sjöstrand, S. Ask, J.R. Christiansen, R. Corke, N. Desai, P. Ilten et al., An introduction to PYTHIA 8.2. Comput. Phys. Commun. 191, 159 (2015). https://doi.org/10.1016/j.cpc.2015.01.024. arXiv: 1410.3012
82. M. Cacciari, G.P. Salam, G. Soyez, The anti- $k_{t}$ jet clustering algorithm. JHEP 04, 063 (2008). https://doi.org/10.1088/1126-6708/ 2008/04/063. arXiv:0802.1189

83. DELPHES 3 collaboration, DELPHES 3 , a modular framework for fast simulation of a generic collider experiment. JHEP 02, 057 (2014). https://doi.org/10.1007/JHEP02(2014)057. arXiv: 1307.6346

84. M. Drees, H. Dreiner, D. Schmeier, J. Tattersall, J.S. Kim, CheckMATE: confronting your favourite new physics model with LHC data. Comput. Phys. Commun. 187, 227 (2015). https://doi.org/10. 1016/j.cpc.2014.10.018. arXiv:1312.2591

85. I. Hinchliffe, F.E. Paige, M.D. Shapiro, J. Soderqvist, W. Yao, Precision SUSY measurements at CERN LHC. Phys. Rev. D 55, 5520 (1997). https://doi.org/10.1103/PhysRevD.55.5520. arXiv:hep-ph/9610544

86. C. Han, L. Wu, J.M. Yang, M. Zhang, Y. Zhang, New approach for detecting a compressed bino/wino at the LHC. Phys. Rev. D 91, 055030 (2015). https://doi.org/10.1103/PhysRevD.91.055030. arXiv: 1409.4533

87. C. Han, A. Kobakhidze, N. Liu, A. Saavedra, L. Wu, J.M. Yang, Probing light higgsinos in natural SUSY from monojet signals at the LHC. JHEP 02, 049 (2014). https://doi.org/10.1007/ JHEP02(2014)049. arXiv:1310.4274

88. H. Baer, A. Mustafayev, X. Tata, Monojet plus soft dilepton signal from light higgsino pair production at LHC14. Phys. Rev. D 90, 115007 (2014). https://doi.org/10.1103/PhysRevD.90.115007. arXiv: 1409.7058

89. A. Barr, J. Scoville, A boost for the EW SUSY hunt: monojetlike search for compressed sleptons at LHC14 with $100 \mathrm{fb}^{\text {? }}$. JHEP 04, 147 (2015). https://doi.org/10.1007/JHEP04(2015)147. arXiv: 1501.02511

90. Z. Han, G.D. Kribs, A. Martin, A. Menon, Hunting quasidegenerate higgsinos. Phys. Rev. D 89, 075007 (2014). https://doi.org/10. 1103/PhysRevD.89.075007. arXiv:1401.1235

91. CMS collaboration, Search for new physics in events with two soft oppositely charged leptons and missing transverse momentum in proton-proton collisions at $\sqrt{s}=13 \mathrm{TeV}$. Phys. Lett. B 782, 440 (2018). https://doi.org/10.1016/j.physletb.2018.05.062. arXiv: 1801.01846

92. ATLAS collaboration, Search for direct stau production in events with two hadronic tau leptons in $\mathrm{s}=13 \mathrm{TeV}$ pp collisions with the ATLAS detector

93. B. Dutta, A. Gurrola, W. Johns, T. Kamon, P. Sheldon, K. Sinha, Vector boson fusion processes as a probe of supersymmetric electroweak sectors at the LHC. Phys. Rev. D 87, 035029 (2013). https://doi.org/10.1103/PhysRevD.87.035029. arXiv:1210.0964

94. K. Albertsson et al., Machine learning in high energy physics community white paper. J. Phys. Conf. Ser. 1085, 022008 (2018). https://doi.org/10.1088/1742-6596/1085/2/022008. arXiv: 1807.02876

95. M. Abdughani, J. Ren, L. Wu, J.M. Yang, J. Zhao, Supervised deep learning in high energy phenomenology: a mini review. Commun. Theor. Phys. 71, 955 (2019). https://doi.org/10.1088/0253-6102/ 71/8/955. arXiv: 1905.06047

96. M. Abdughani, J. Ren, L. Wu, J.M. Yang, Probing stop pair production at the LHC with graph neural networks. JHEP 08, 055 (2019). https://doi.org/10.1007/JHEP08(2019)055. arXiv:1807.09088

97. J. Ren, L. Wu, J.M. Yang, J. Zhao, Exploring supersymmetry with machine learning. Nucl. Phys. B 943, 114613 (2019). https://doi. org/10.1016/j.nuclphysb.2019.114613. arXiv:1708.06615

98. S. Caron, J.S. Kim, K. Rolbiecki, R. Ruiz de Austri, B. Stienen, The BSM-AI project: SUSY-AI-generalizing LHC limits on supersymmetry with machine learning. Eur. Phys. J. C 77, 257 (2017). https:// doi.org/10.1140/epjc/s10052-017-4814-9. arXiv:1605.02797 